\title{
Valuación de opciones con ajustes a distribuciones $\alpha$-estables y contabilidad bajo la norma internacional de información financiera
}

\author{
Options pricing with $\alpha$-stable distributions fits and \\ accounting under international financial reporting standard
}

\author{
José Antonio Climent Hernández ${ }^{* 1}$, Itzel Gómez Pinto \\ ${ }^{1}$ Universidad Autónoma Metropolitana, México \\ ${ }^{2}$ Universidad Juárez Autónoma de Tabasco, México
}

Recibido el 7 de marzo de 2019; aceptado el 13 de abril de 2020

Disponible en Internet el: 9 de mayo de 2020

\section{Resumen}

En este trabajo se pretende analizar los rendimientos del dólar estadounidense, euro, libra esterlina y yen, con el peso mexicano, son estimados los estadísticos descriptivos y los parámetros $\alpha$-estables, las pruebas de bondad de ajuste justifican estadísticamente la idoneidad de las distribuciones $\alpha$-estables para modelar el comportamiento de los rendimientos, también son estimados los exponentes de autosimilitud y los índices de memoria, la valuación de las opciones europeas de compra y de venta es realizada con el modelo gaussiano y con el modelo $\alpha$-estable, y la contabilización es presentada bajo la norma internacional de información financiera concluyendo que las opciones $\alpha$-estables cuantifican más adecuadamente el riesgo de tipo de cambio que las opciones gaussianas, recomendando realizar un análisis para minimizar las pérdidas potenciales derivadas de las obligaciones económicas adquiridas

\footnotetext{
*Autor para correspondencia

Correo electrónico jach@azc.uam.mx (J.A. Climent Hernández).

La revisión por pares es responsabilidad de la Universidad Nacional Autónoma de México.

http://dx.doi.org/10.22201/fca.24488410e.2021.2491

0186- 1042/@ 2019 Universidad Nacional Autónoma de México, Facultad de Contaduría y Administración. Este es un artículo Open Access bajo la licencia CC BY-NC-SA (https://creativecommons.org/licenses/by-nc-sa/4.0/)
} 
por la emisión de opciones y que la norma internacional de información financiera alinea los objetivos de gestión de riesgos para reflejar las actividades transmitiendo el objetivo y el efecto de las opciones.

Código JEL: C16, C46, C14, D81, G12, G13

Palabras clave: Procesos estocásticos $\alpha$-estables; Ingeniería financiera; Normas internacionales de información financiera

\begin{abstract}
This paper pretends to analyze the returns of US dollar, euro, sterling and yen, with the Mexican peso, descriptive statistics and $\alpha$-stable parameters are estimated, goodness of fit tests statistically justify the suitability of $\alpha$-stable distributions to model the returns of currencies, the self-similarity exponents and memory indices are also estimated, the European call and put option's pricing is done with the Gaussian model and with the $\alpha$-stable model, and the accounting is presented under international financial reporting standard, concluding that the $\alpha$-stable model quantify more adequately the exchange rate risk than the Gaussian model, recommending an analysis to minimize the potential losses arising from the economic obligations acquired for issuing options and that international financial reporting standard is aligning the risk management objectives to reflects the risk management activities and transmitting the goal and effect of the options.
\end{abstract}

JEL Code: C16, C46, C14, D81, G12, G13

Keywords: Stochastic processes $\alpha$-stables; Finance engineering; International financial reporting standard

\title{
Introducción
}

La ingeniería financiera modela con procesos estocásticos $\alpha$-estables los rendimientos de productos financieros que presentan impactos financieros y económicos de magnitudes relevantes por los valores extremos y la asimetría de los rendimientos. Los mercados financieros han evolucionado con las tecnologías de la información, la competencia global, la ingeniería financiera y la administración de riesgos que han innovado en la estructura de productos para satisfacer las necesidades de inversionistas y emisores. En el contexto de la valuación de opciones, Bachelier (1900) aplicó un proceso estocástico gaussiano para modelar precios de productos financieros de la bolsa de París. Kendall y Hill (1953) y Kruizenga (1956) realizaron un análisis empírico y rechazaron la capacidad del proceso estocástico gaussiano para modelar los precios de las acciones. Osborne (1959) justificó la pertinencia del proceso estocástico gaussiano para modelar rendimientos. Osborne (1959), Sprenkle (1961), Boness (1964), y Samuelson (1965) aplicaron un proceso gaussiano para valuar opciones. Black y 
Scholes (1973), y Merton (1973) modelaron los rendimientos como un proceso estocástico gaussiano y presentaron un modelo para valuar opciones.

Los precios de los productos derivados están determinados por la distribución de los rendimientos, entonces, la valuación de opciones es obtenida al maximizar el valor presente de la esperanza condicional del pago contingente en función de la medida neutral al riesgo, como lo propusieron Cox y Ross (1976) y Ross (1976), por lo tanto, la estimación de parámetros de la distribución de los rendimientos determina los precios de las opciones sobre productos financieros.

Merton (1976) propuso un modelo donde los rendimientos evolucionan como procesos estocásticos con discontinuidades donde los cambios están compuestos por dos factores: cambios moderados e independientes que son modelados con el proceso estocástico gaussiano y cambios ocasionales de magnitud superior que son modelados con un proceso estocástico de Poisson compuesto. El modelo de Merton (1976) es un proceso de difusión con saltos gaussianos y si la intensidad de los saltos es nula, entonces, es obtenido el modelo de Black y Scholes (1973), que es un caso particular del modelo de Merton (1976). Ambos modelos son procesos estocásticos de Lévy (1937).

Sierra Juárez (2007) aplicó un proceso estocástico gaussiano fraccionario para modelar la evolución de paridades e índices. Valúo opciones en mercados fraccionarios donde el proceso estocástico gaussiano fraccionario es un caso particular de los procesos estocásticos $\alpha$-estables. Los modelos para valuar opciones ajustan los rendimientos a procesos estocásticos para fundamentar el comportamiento empírico con distribuciones de probabilidad y los procesos estocásticos de Lévy han sido exitosos. Esscher (1932) propuso una medida de probabilidad equivalente que conserva las propiedades de los procesos estocásticos de Lévy. La transformada de Girsanov (1960) para procesos estocásticos con saltos es un caso particular de la transformada de Esscher. Itô (1942) planteó la descomposición Lévy-Itô que asegura que los procesos estocásticos de Lévy son la suma de un movimiento browniano con tendencia y una serie de procesos de Poisson compuestos e independientes.

Mandelbrot (1963) propuso un proceso $\alpha$-estable simétrico para modelar los precios del algodón. Fama (1963, 1965a, 1965b) y Mandelbrot y Taylor (1967) rechazaron el supuesto gaussiano y propusieron procesos estocásticos $\alpha$-estables. McCulloch (1978, 1985, 1987, 1996) modeló los rendimientos como procesos $\alpha$-estables simétricos y propuso valuar opciones con distribuciones $\alpha$-estables simétricas. Janicki et al. (1997), Popova y Ritchken (1998), y Hurst, Platen y Rachev (1999) desarrollaron modelos para valuar opciones sobre subyacentes con rendimientos que presentan distribuciones $\alpha$-estables simétricas. Carr y Wu (2003) propusieron un modelo de momentos finitos suponiendo que la medida neutral al riesgo 
tiene sesgo negativo máximo y aplicaron procesos estocásticos $\alpha$-estables porque conservan la forma sobre escala y son adecuados para modelar la estructura de la sonrisa de volatilidad implícita que captura los cambios de la volatilidad originados por cambios ocasionales de magnitud superior a través de los extremos de la distribución.

McCulloch (2003) formuló un modelo para valuar opciones aplicando la transformada de Esscher (1932) como la convolución de una distribución $\alpha$-estable extrema negativa y una distribución $\alpha$-estable extrema positiva ajustada exponencialmente.

En México, Contreras Piedragil y Venegas Martínez (2011), Climent Hernández y Venegas Martínez (2013), Rodríguez Aguilar y Cruz Aké (2013), y Climent Hernández y Cruz Matú (2017) han presentado investigaciones donde son valuadas opciones con distribuciones $\alpha$-estables, probando estadísticamente la idoneidad e indicando las diferencias con el modelo gaussiano.

La volatilidad subyacente es un factor que tiene influencia significativa en la valuación de opciones y evoluciona de forma aleatoria, entonces, es posible modelar con procesos estocásticos, por ejemplo: Hull y White (1987) modelaron la volatilidad como un proceso log-gaussiano sin reversión a la media. Scott (1987) modeló la volatilidad como un proceso estocástico con reversión a la media. Stein y Stein (1991) supusieron que la volatilidad no está correlacionada con el precio subyacente y no captura los efectos de asimetría de la correlación. Heston (1993) propuso un modelo donde el precio subyacente está modelado por un proceso estocástico log-gaussiano, la volatilidad está modelada con un proceso estocástico Uhlenbeck y Ornstein (1930) con reversión a la media donde el precio y la volatilidad están correlacionados. Heston (1993) obtuvo una fórmula para valuar opciones europeas de compra. Venegas Martínez (2005) desarrolló un modelo bayesiano para valuar productos derivados con información a priori sobre la volatilidad, como valores esperados, y presentó fórmulas aproximadas para valuar opciones de compra con aproximaciones asintóticas y polinómicas de funciones de Bessel.

La valuación y determinación del ejercicio anticipado óptimo de las opciones americanas consiste en determinar la frontera óptima para maximizar el flujo de efectivo por el ejercicio anticipado. Actualmente existen métodos de aproximación como el numérico de Villeneuve y Zanette (2002), el de diferencias finitas de Brennan y Schwartz (1977), y una fórmula analítica aproximada de Barone Adesi y Whaley (1987). Cox et al. (1979) modelaron los precios como un proceso estocástico binomial y presentaron un modelo para valuar opciones en tiempo discreto que converge al modelo de Black y Scholes (1973) con opciones europeas y permite la valuación de opciones americanas de venta considerando los flujos de efectivo por el ejercicio anticipado. Climent Hernández (2014) estimó los parámetros de la probabi- 
lidad libre de riesgo y aplicó la programación dinámica estocástica para modelar el precio subyacente como un proceso binomial que modela las tasas de interés y la volatilidad como funciones deterministas o como procesos estocásticos e incluyó el pago de dividendos, presentó la diferencia con el modelo de Cox et al. (1979) y la convergencia al modelo propuesto por Barone Adesi y Whaley (1987).

Martínez Palacios et al. (2012) indican que el modelo de Cox et al. (1979) y el modelo de Climent Hernández (2014) convergen, mediante un enfoque de control óptimo estocástico, a una opción europea con el modelo de Black y Scholes (1973) más un valor positivo que satisface otras condiciones, y como se ha mostrado el valor se aproxima al modelo Barone Adesi y Whaley (1987).

En el contexto de la norma internacional de información financiera (NIIF), Landeros Olascoaga (2008) indica que los productos financieros de deuda o de capital con plazo menor que un año y que cotizan en el mercado de valores y las inversiones para negociación o venta están clasificados como inversiones temporales y son presentadas en el activo circulante. Morales Díaz (2012) analiza la contabilidad de cobertura utilizando opciones, contextualiza las normas de contabilidad de coberturas, estudia en qué caso un portafolio de opciones deja de ser un producto de cobertura, trata el problema del valor de las opciones en el tiempo y la contabilidad de los flujos de efectivo, y presenta los cambios que introduce la NIIF en las coberturas con opciones. Pelmeneva y Talipova (2015) indican que los procesos de globalización e integración de las empresas en la economía mundial requieren unificación de la información financiera, transparencia, uniformidad y procedimientos de cálculos financieros porque la NIIF indica cómo se tienen que generar los informes financieros, imponiendo obligaciones en el procedimiento de presentación. Glover y Werner (2015) proporcionan una plantilla para la enseñanza de la NIIF en los planes de estudios contables, identifican instrucciones para enseñar la NIIF, presentan recomendaciones para mejorar las prácticas contables, indican que las encuestas muestran que la integración de la NIIF en las aulas no coincide con las expectativas internacionales de la contabilidad, las firmas de contabilidad esperan que los candidatos conozcan los estándares internacionales de contabilidad para adoptar la NIIF. Mantilla (2016) indica que la valuación retrospectiva de la efectividad de la cobertura no es requerida, la NIIF emitida en el año 2014 reemplaza a las versiones anteriores y es obligatoria para los períodos que comienzan en o después del 1 de enero de 2018, la presentación de estados financieros requiere que las pérdidas y las ganancias sean presentadas en un elemento separado en el estado de resultados, y la contabilidad de cobertura permite que en los estados financieros sean reflejadas las actividades de administración del riesgo. Gómez Pinto et al. (2019) presentan los cálculos para la contabilidad de productos derivados 
con fundamento en la NIIF, indican que la NIIF-9 es para revelar los requerimientos para la clasificación y medición de los activos financieros y la contabilidad de cobertura, indican que en el estado de resultados son registrados los cambios periódicos de las valuaciones del activo o del pasivo y que el valor de los productos derivados es clasificado en el balance general como un activo o pasivo, dependiendo de los derechos o las obligaciones establecidas en el contrato, en el caso de opciones, los derechos u obligaciones concluyen en la fecha de vencimiento y la operación es liquidada.

Los objetivos del trabajo son modelar la evolución de los rendimientos con procesos estocásticos $\alpha$-estables para valuar opciones europeas de compra sobre las paridades de los tipos de cambio del dólar estadounidense, euro, libra esterlina y yen con el peso mexicano estimando los parámetros de la distribución de los rendimientos con del método de máxima verosimilitud para justificar la pertinencia con de las pruebas de bondad de ajuste Kolmogorov y Smirnov (KS) y Anderson y Darling (AD) para administrar riesgos financieros más adecuadamente, innovando con la estimación de los índices de memoria y funciones de riesgo y realizar la contabilidad de las opciones gaussianas y $\alpha$-estables bajo la NIIF.

El trabajo está organizado de la forma siguiente: en la sección 2 es presentado un análisis del desempeño de las paridades, la estimación de los estadísticos descriptivos de los rendimientos, la estimación de los parámetros $\alpha$-estables, las pruebas de bondad de ajuste a las distribuciones $\alpha$-estables, un análisis cualitativo del ajuste de los rendimientos a las distribuciones gaussianas y $\alpha$-estables, la estimación de los exponentes de autosimilitud, los índices de memoria y las funciones de riesgo. En la sección 3 es realizada la valuación de las opciones europeas de compra y de venta. En la sección 4 es realizada la contabilidad de las opciones europeas gaussianas y $\alpha$-estables con la NIIF-9. En la sección 5 son presentadas las conclusiones, y por último son presentadas las referencias bibliográficas.

\section{Análisis de las paridades de los tipos de cambio}

Las trayectorias de las paridades de los tipos de cambio del dólar, euro, libra esterlina y yen durante el periodo hábil del 3 de enero de 2011 al 26 de julio de 2018 son presentadas en la figura 1. 


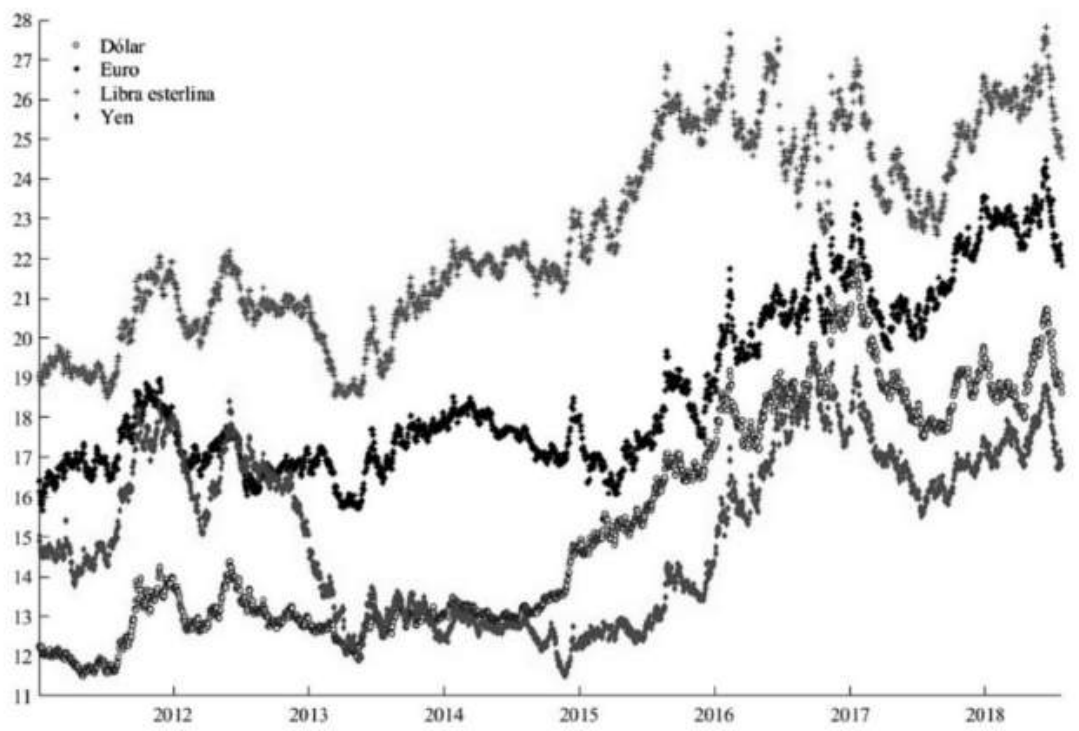

Figura 1. Trayectorias de las paridades de los tipos de cambio Fuente: Elaboración propia con datos del Banco de México

La figura 1 presenta las trayectorias del dólar (circunferencia), euro (asterisco), libra esterlina (cruz) y yen multiplicado por cien pesos (rombo) con 1,901 paridades diarias para cada tipo de cambio. Los acontecimientos estilizados indican que las paridades presentan asimetría positiva y leptocurtosis. Los estadísticos descriptivos de las primeras 1,882 paridades son presentadas en la tabla 1.

Tabla 1

Estadísticos descriptivos de las paridades de los tipos de cambio

\begin{tabular}{lllllll}
\hline Paridad & Mínimo & Máximo & Promedio & Desviación & Asimetría & Curtosis \\
\hline Dólar & 11.5023 & 21.9076 & 15.2903 & 2.8105 & 0.4608 & 1.7124 \\
Euro & 15.6622 & 24.4712 & 18.6619 & 2.1480 & 0.7887 & 2.3505 \\
Libra & 18.5007 & 27.7979 & 22.6218 & 2.4146 & 0.1667 & 1.8278 \\
Yen & 0.1152 & 0.1981 & 0.1518 & 0.0209 & 0.0368 & 1.6083 \\
\hline
\end{tabular}

Fuente: Elaboración propia con datos del Banco de México 
La tabla 1 presenta rangos, promedios, desviaciones estándar, coeficientes de asimetría y curtosis. El dólar presenta la mayor volatilidad, seguido por la libra esterlina, el euro y el yen. El dólar presenta el mayor coeficiente de variación ${ }^{1}$ (0.1838), seguido por el yen (0.1378), el euro (0.1151), y la libra esterlina (0.1067) confirmando los acontecimientos estilizados identificados en la figura 1.

\section{Análisis de los rendimientos}

Los 1,881 rendimientos diarios de los tipos de cambio son presentados en la figura 2.

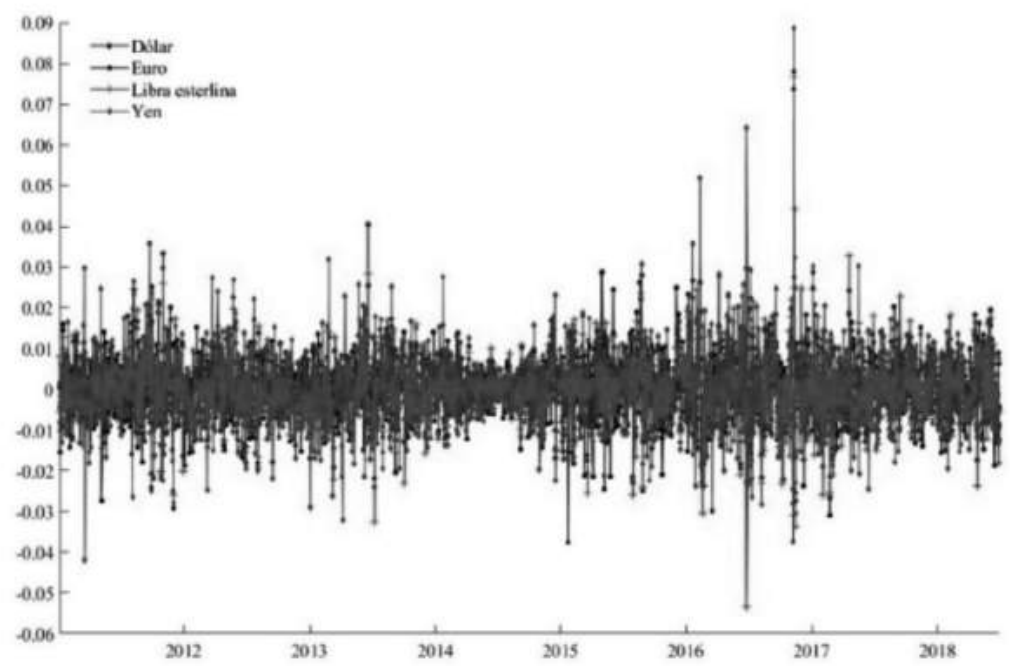

Figura 2. Rendimientos diarios de las paridades de los tipos de cambio

Fuente: Elaboración propia con datos del Banco de México

La Figura 2 presenta los rendimientos diarios de las paridades de los tipos de cambio con 1,881 observaciones con cúmulos de volatilidad elevada que representan cambios relevantes durante periodos cortos y cúmulos de volatilidad moderada que representan cambios moderados en periodos más extensos que los periodos de cambios relevantes. La estimación de los estadísticos descriptivos de los rendimientos de las divisas es presentada en la tabla 2.

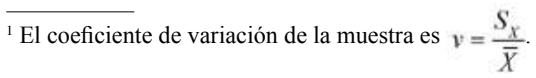


Tabla 2

Estadísticos descriptivos de los rendimientos de las paridades de los tipos de cambio

\begin{tabular}{lllllll}
\hline Paridad & Mínimo & Máximo & Promedio & Desviación & Asimetría & Curtosis \\
\hline Dólar & -0.029854 & 0.073724 & 0.000252 & 0.007054 & 0.795047 & 11.148338 \\
Euro & -0.037420 & 0.078107 & 0.000178 & 0.007742 & 0.482035 & 9.463255 \\
Libra & -0.053581 & 0.076503 & 0.000165 & 0.007719 & 0.299183 & 10.785422 \\
Yen & -0.041742 & 0.088755 & 0.000090 & 0.009428 & 0.745728 & 9.394673 \\
\hline
\end{tabular}

Fuente: Elaboración propia con datos del Banco de México

La tabla 2 presenta rangos, promedios, desviaciones estándar, coeficientes de asimetría y curtosis de los rendimientos de las paridades. Los rendimientos presentan promedios positivos. Los rendimientos del yen presentan la mayor desviación estándar, seguidos por el euro, la libra esterlina y el dólar. Los rendimientos presentan coeficientes de asimetría positivos, entonces, los rendimientos presentan distribuciones que se extienden hacia valores positivos con mayor frecuencia que a valores negativos. Los coeficientes de curtosis indican que las distribuciones son leptocúrticas. Por lo tanto, los rendimientos de las divisas presentan distribuciones asimétricas y leptocúrticas con respecto a la distribución gaussiana. Las probabilidades gaussianas y empíricas de los rendimientos de las divisas son presentadas en la figura 3.

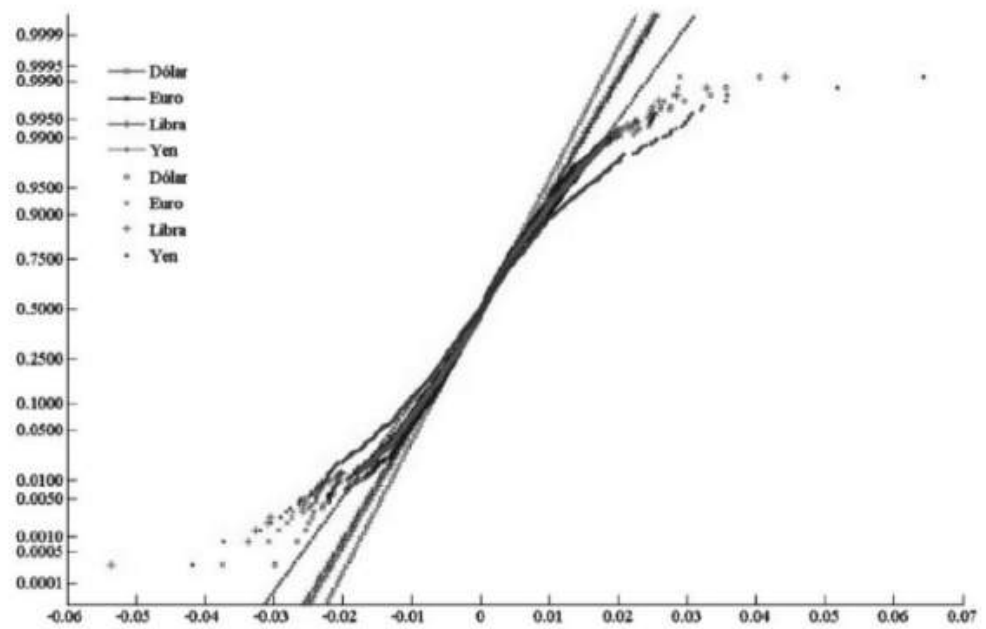

Figura 3. Probabilidades gaussianas y probabilidades de los rendimientos de las divisas Fuente: Elaboración propia con datos del Banco de México 
La figura 3 presenta las probabilidades gaussianas (líneas rectas con símbolos) en función de los rendimientos de las divisas: dólar (circunferencias), euro (equis), libra esterlina (cruces) y yen (rombos) y las probabilidades empíricas de los rendimientos de las divisas (símbolos) en función de los rendimientos de las divisas. Confirmando que los rendimientos presentan asimetría y eventos extremos en las distribuciones de los rendimientos. Los cuantiles de los rendimientos gaussianos y empíricos de las divisas son presentados en la figura 4.

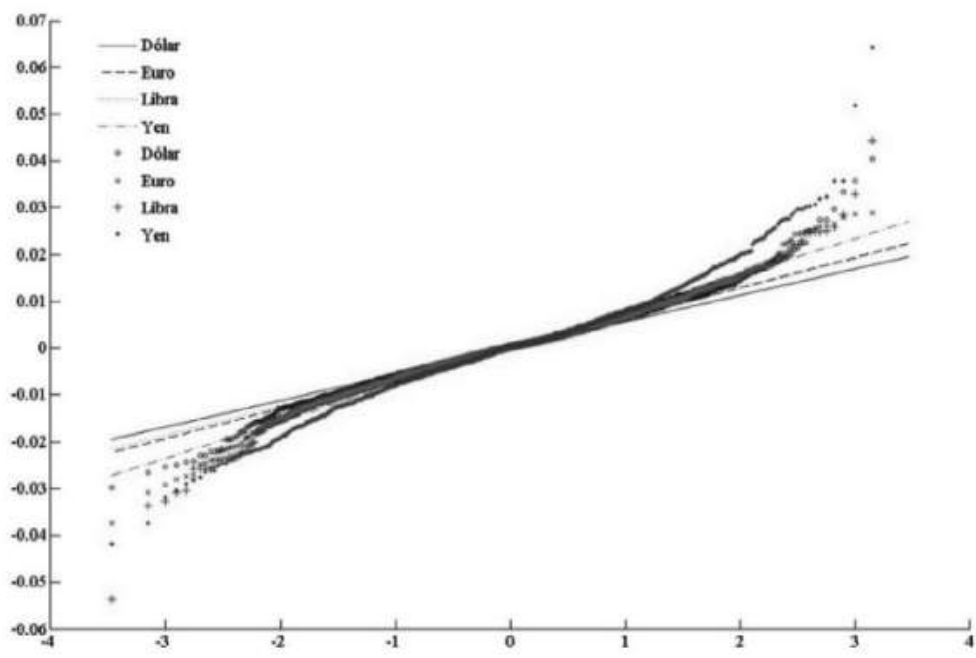

Figura 4. Cuantiles gaussianos y cuantiles de las divisas Fuente: Elaboración propia con datos del Banco de México

La figura 4 presenta los cuantiles gaussianos (líneas) en función de los cuantiles gaussianos de las divisas: dólar (línea continua), euro (línea discontinua), libra esterlina (línea punteada) y yen (línea discontinua y punteada) y los cuantiles de las divisas (símbolos) en función de los cuantiles gaussianos de las divisas: dólar (circunferencias), euro (equis), libra esterlina (cruces) y yen (rombos). Confirmando que los rendimientos presentan asimetría y leptocurtosis en las distribuciones de los rendimientos, entonces, las distribuciones gaussianas están subestimando pérdidas y ganancias relevantes. Por lo tanto, los inversionistas que no consideran la asimetría y la leptocurtosis bajo el supuesto gaussiano están expuestos a riesgos que no están considerando.

El análisis cualitativo indica que los rendimientos no presentan distribuciones gaussianas, entonces, los rendimientos son ajustados a distribuciones $\alpha$-estables para modelar caracterís- 
ticas de asimetría y leptocurtosis para cuantificar más adecuadamente eventos relevantes que están considerados inadecuadamente por la distribución gaussiana y que representan riesgos para los inversionistas por la falta de un modelo congruente con los rendimientos empíricos de las divisas.

\section{Estimación de los parámetros $\alpha$-estables}

Las distribuciones $\alpha$-estables están caracterizadas por cuatro parámetros y generalmente son denotadas por $S(\alpha, \beta, \gamma, \delta)$. El parámetro de estabilidad $0<\alpha \leq 2$ determina el grado de leptocurtosis y la pendiente con la que decrecen los extremos de la distribución. El parámetro de asimetría $-1 \leq \beta \leq 1$ define el grado de asimetría de la distribución. El parámetro de escala $\gamma>0$ indica las unidades de dispersión que tiene la distribución con respecto al parámetro de localización. El parámetro de localización $-\infty<\delta<\infty$ determina el punto de localización que tiene la distribución. Las distribuciones $\alpha$-estables, en general, no tienen una expresión analítica cerrada para caracterizar a la variable aleatoria, pero con la función característica $\varphi_{Z}(\kappa)$ o con la función de cumulantes $\psi_{Z}(\kappa)$ es posible caracterizar de forma única a cualquier variable aleatoria $\alpha$-estable. Una variable aleatoria $Y$ es $\alpha$-estable si y sólo si $Y=\gamma Z+\delta$, donde $Z$ es una variable aleatoria con la función característica:

$$
\varphi_{z}(\kappa)=E(\exp (\imath \kappa Z))=\left\{\begin{array}{l}
\exp \left(-|\kappa|^{\alpha}\left(1-\imath \beta \operatorname{sgn}(\kappa) \tan \left(\frac{\pi \alpha}{2}\right)\right)\right) \\
\exp \left(-|\kappa|\left(1-\imath \frac{2}{\pi} \beta \sin (\kappa) \ln (\kappa)\right)\right) \quad \text { si } \alpha=1,
\end{array}\right.
$$

donde:

$$
\operatorname{sgn}(\kappa)=\left\{\begin{array}{cc}
\frac{\kappa}{|\kappa|} & \kappa \neq 0, \\
0 & \kappa=0 .
\end{array}\right.
$$

La función de cumulantes de la variable aleatoria $Z$ es:

$$
\psi_{Z}(\kappa)=\ln \left(\varphi_{Z}(\kappa)\right)= \begin{cases}-|\kappa|^{\alpha}(1-\imath \beta \operatorname{sgn}(\kappa) \tan (\theta)) & \text { si } \alpha \neq 1, \\ -|\kappa|\left(1-\frac{2 l}{\pi} \beta \operatorname{sgn}(\kappa) \ln (\kappa)\right) & \text { si } \alpha=1,\end{cases}
$$


donde $t^{2}=-1$. Las distribuciones $\alpha$-estables tienen expresiones analíticas cerradas para los casos siguientes: Gauss: $S\left(2,0,2^{-1} \sigma, \mu\right)$, Cauchy: $S(1,0, \gamma, \delta)$ y Lévy: $S\left(2^{-1}, \pm 1, \gamma, \delta\right)$.

$\mathrm{El}$ análisis de los rendimientos indica que las distribuciones son asimétricas y leptocúrticas, entonces, la estimación de parámetros $\alpha$-estables es realizada con del método de máxima verosimilitud y parametrización 1 . La estimación de los parámetros $\alpha$-estables es presentada en la tabla 3.

Tabla 3

Estimación de los parámetros $\alpha$-estables al 95\% de confianza

\begin{tabular}{lcccc}
\hline Paridad & $\alpha$ & $\beta$ & $\gamma$ & $\delta$ \\
\hline Dólar & $1.7793 \pm 0.0639$ & $0.1436 \pm 0.2386$ & $0.004195 \pm 0.000173$ & $0.000257 \pm 0.000331$ \\
Euro & $1.8259 \pm 0.0601$ & $0.0000 \pm 0.2968$ & $0.004809 \pm 0.000193$ & $0.000152 \pm 0.000376$ \\
Libra & $1.7832 \pm 0.0641$ & $0.0000 \pm 0.2467$ & $0.004587 \pm 0.000190$ & $0.000186 \pm 0.000362$ \\
Yen & $1.7862 \pm 0.0634$ & $0.1605 \pm 0.2421$ & $0.005694 \pm 0.000234$ & $0.000066 \pm 0.000449$ \\
\hline
\end{tabular}

Fuente: Elaboración propia con datos del Banco de México

La tabla 3 presenta las estimaciones de los parámetros $\alpha$-estables. Los parámetros de estabilidad y de asimetría son consistentes con las estimaciones presentadas internacionalmente por Dostoglou y Rachev (1999), Ortobelli et al. (2002), Ortobelli et al. (2004), Rachev et al. (2004), Čížek et al. (2005), Ortobelli et al. (2005), Scalas y Kim (2006), y nacionalmente por Contreras Piedragil y Venegas Martínez (2011), Climent Hernández y Venegas Martínez (2013), Climent Hernández y Cruz Matú (2017), y Climent Hernández et al. (2017). Los parámetros de escala indican que la mayor dispersión la presentan el yen, seguidos por el euro, la libra y el dólar. Por lo tanto, los rendimientos del dólar y el yen presentan asimetría positiva y leptocurtosis, y los rendimientos del euro y la libra esterlina presentan únicamente leptocurtosis.

\section{Prueba de bondad de ajuste Kolmogorov y Smirnov}

El análisis para probar la hipótesis de que los rendimientos presentan distribuciones gaussianas o $\alpha$-estables con el estadístico de bondad de ajuste KS es presentado en la tabla 4. 
Tabla 4

Pruebas KS gaussianas al 99\% de confianza

\begin{tabular}{lllll}
\hline Paridad & $D$ & $\mathrm{P}(D>d)$ & $D_{\alpha}$ & $\mathrm{P}_{\alpha}(D>d)$ \\
\hline Dólar & 0.0552 & 0.0000 & 0.0195 & 0.4675 \\
Euro & 0.0456 & 0.0008 & 0.0187 & 0.5256 \\
Libra & 0.0475 & 0.0004 & 0.0196 & 0.4632 \\
Yen & 0.0496 & 0.0002 & 0.0271 & 0.1231 \\
\hline
\end{tabular}

Fuente: Elaboración propia con datos del Banco de México

La tabla 4 presenta los estadísticos $D$ para los rendimientos de las divisas y los niveles de significación descriptivos, rechazando las hipótesis de que los rendimientos presentan distribuciones gaussianas, y no rechazando las hipótesis de que los rendimientos presentan distribuciones $\alpha$-estables. Las pruebas KS indican que los rendimientos empíricos presentan eventos extremos y que las distribuciones $\alpha$-estables modelan la asimetría y la leptocurtosis más adecuadamente que la distribución gaussiana, permitiendo cuantificar los riesgos de mercado más apropiadamente. Las pruebas de bondad de ajuste KS son complementadas con los estadísticos AD para justificar la pertinencia de las hipótesis gaussianas y los ajustes a las distribuciones $\alpha$-estables.

\section{Prueba de bondad de ajuste Anderson y Darling}

El análisis cuantitativo para probar la hipótesis de que los rendimientos presentan distribuciones gaussianas o $\alpha$-estables es realizado con del estadístico de bondad de ajuste AD que es presentado en la tabla 5 .

\section{Tabla 5}

Pruebas AD gaussianas al 99\% de confianza

\begin{tabular}{lllll}
\hline Paridad & $A^{2}$ & $\mathrm{P}\left(A^{2}>a^{2}\right)$ & $A_{\alpha}^{2}$ & $\mathrm{P}_{\alpha}(D>d)$ \\
\hline Dólar & $\infty$ & 0.0000 & 0.8210 & 0.4661 \\
Euro & $\infty$ & 0.0000 & 0.6751 & 0.5799 \\
Libra & $\infty$ & 0.0000 & 0.6889 & 0.5682 \\
Yen & 49.0196 & 0.0000 & 0.5891 & 0.6583 \\
\hline
\end{tabular}

Fuente: Elaboración propia con datos del Banco de México 
La tabla 5 presenta los estadísticos $A^{2}$ para los rendimientos de las divisas y los niveles de significación descriptivos, rechazando las hipótesis de que los rendimientos presentan distribuciones gaussianas, y no rechazando las hipótesis de que los rendimientos presentan distribuciones $\alpha$-estables. Las pruebas AD indican que los rendimientos empíricos presentan eventos extremos que las distribuciones $\alpha$-estables modelan más adecuadamente que la distribución gaussiana, lo que permite administrar riesgos de mercado con mayor efectividad para la toma de decisiones.

Los resultados de las tablas 4 y 5 indican que las distribuciones $\alpha$-estables son más eficientes para modelar el comportamiento empírico de los rendimientos y administrar riesgos de tipo de cambio con mayor efectividad que con la distribución gaussiana. Por lo tanto, y de acuerdo con las pruebas de bondad de ajuste $\mathrm{KS}$ y $\mathrm{AD}$, las distribuciones $\alpha$-estables son idóneas para modelar los rendimientos de las paridades en el periodo estudiado y es posible valuar opciones sobre los tipos de cambio.

\section{Ajustes de las distribuciones}

Los ajustes de las distribuciones gaussiana y $\alpha$-estable a los rendimientos del dólar, euro, libra esterlina y yen son presentados en la figura 5 .
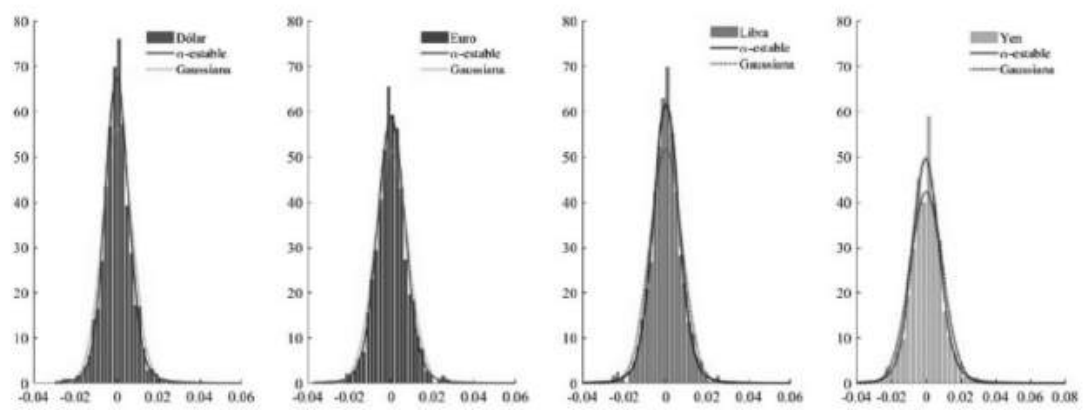

Figura 5. Ajuste de la distribución $\alpha$-estable a los rendimientos de las divisas Fuente: Elaboración propia con datos del Banco de México

La figura 5 presenta los ajustes de las distribuciones gaussiana y $\alpha$-estable a los rendimientos de las divisas. Confirmando que las propiedades de asimetría y leptocurtosis están modeladas más adecuadamente por las distribuciones $\alpha$-estables que por las distribuciones gaussianas. Por lo tanto, los resultados de las pruebas de bondad de ajuste y los ajustes presentados en la 
figura 5 muestran que las distribuciones $\alpha$-estables son eficientes para modelar el comportamiento de los rendimientos y cuantificar los riesgos de los tipos de cambio.

\section{Estimación de los exponentes de autosimilitud}

El proceso $X(t)$ es autosimilar con exponente $H>0$, si para toda $a \in(0, \infty)$, las distribuciones finito dimensionales de $X(a t)$ son idénticas a las distribuciones finito dimensionales de $a^{H} X(t)$ :

$$
\left(X\left(a t_{1}\right), \quad, X\left(a t_{n}\right)\right) \underline{\underline{d}}\left(a^{H} X\left(t_{1}\right), a^{H} X\left(t_{n}\right)\right)
$$

El movimiento $\alpha$-estable simétrico de Lévy (MES) es autosimilar con $H=\alpha^{-1}$, por lo tanto, el exponente de autosimilitud $H \in\left[2^{-1}, \infty\right)$, es decir, el MB es autosimilar con $H=2^{-1}$.

Belov et al. (2006) indican que los procesos $\alpha$-estables son una herramienta potente y versátil para los modelos financieros. Demuestran la eficiencia de los parámetros $\alpha$-estables estimados por el método de máxima verosimilitud. Realizan pruebas de hipótesis para autosimilitud y multifractalidad. Estiman el exponente de Hurst en el dominio de tiempo con los métodos momentos absolutos (MA), convergencia de la varianza (CV), rango reescalado (RR) y varianza de los residuales (VR), y en el dominio de frecuencia utilizan los métodos periodograma (PG), Whittle y Abry Veitch (WAV).

Barunik y Kristoufek (2010) muestran que las propiedades en la estimación del exponente de Hurst cambian con la presencia de leptocurtosis. Realizan simulaciones Monte Carlo para analizar RR, análisis de fluctuación sin tendencia multifraccionaria (AFSTMF), media móvil sin tendencia (MMST) y exponente de Hurst generalizado (EHG). Estiman el exponente de Hurst a partir de series independientes con diferentes parámetros de estabilidad. Indican que el método EHG proporciona la menor varianza y el menor sesgo con respecto a otros métodos. También indican que se ha demostrado que el EHG es $H(q) \approx q^{-1}$ para $q>\alpha$ y que $H(q) \approx \alpha^{-1}$ para $q \leq \alpha$. Indican que el EHG es adecuado para la detección multifraccionaria, y es comparable con RR, MMST y $\operatorname{AFSTMF(2).~Si~} q=1$, entonces, $H(1)$ está caracterizando la escala de las desviaciones absolutas del proceso. Los métodos de EHG(1) y AFSTMF(1) presentan $\mathrm{E}(H)=\alpha^{-1}$, por lo tanto, $\mathrm{EHG}(1)$ presenta el mejor comportamiento para muestras finitas de entre todos los métodos, con la menor varianza, el menor sesgo y los intervalos de confianza más estrechos. Concluyen que RR y EHG son robustos, EHG $(q)$ supera a todos los métodos mencionados. Por lo tanto, $\operatorname{EHG}(q)$ es útil porque presenta propiedades satisfactorias superando a los otros métodos. 
Climent Hernández et al. (2017) estiman el par ordenado $(\alpha, H)$ para conocer las formas distribuciones $\alpha$-estables, las dimensiones faccionarias de los espacios de probabilidad $(\Omega, F, P)$, las dimensiones fraccionarias de las series de tiempo, los efectos de antipersistencia, independencia estocástica o persistencia, y los procesos estocásticos con los que es posible modelar adecuadamente las series de tiempo de las paridades del dólar, euro, yen y dólar canadiense empleando EHG(1) para estimar los exponentes de autosimilitud $\mathrm{H}$ y realizando las pruebas $t$ y $F$, descartando que las series de las paridades son multifraccionarias.

La estimación del exponente de autosimilitud propuesta por Climent Hernández et al. (2017) es realizada por el método $\mathrm{EHG}(1)$ donde $\mathrm{E}(H)=\alpha^{-1}$ es el límite entre antipersistencia (reversión a la media) y persistencia (memoria larga) para los procesos $\alpha$-estables. El método consiste en realizar 19 particiones $K_{q}(\tau)$ de los rendimientos, donde $t=1, \quad, 19$, entonces:

$$
K_{q}(\tau)=\frac{\sum_{t=1}^{v}|X(t+\tau)-X(t)|^{q}}{\sum_{t=1}^{v}|X(t)|^{q}}
$$

donde $K_{q}(\tau)$ es el estadístico de escala, $q=1$, 10, es el momento, $\tau$ es el incremento entre de los rendimientos acumulados, $n$ es el tamaño de la serie de tiempo, $v=\left\lceil\tau^{-1} n\right\rceil$ es el tamaño de la partición, $X(t)$ es el rendimiento acumulado en el instante $t, \mathrm{y}\lceil\cdot\rceil$ es la función entero superior, entones, $K_{q}(\tau) \approx c \tau^{q H(q)}$. Con los 19 datos es calculada la regresión lineal y es obtenido el primer estimador $\operatorname{EHG}(q)$, después son realizadas 15 regresiones para $\tau=5$, , 19, y calculando el promedio aritmético es obtenido EHG(1). Para el análisis multifractal son considerados los diez primeros momentos $q=1,, 10$ y es realizado el análisis de regresión. Si es lineal, entonces, la serie es autosimilar, y en caso contrario la serie es multifraccionaria.

Los procesos autosimilares son invariantes en distribución bajo escala de tiempo y espacio, las estimaciones de los exponentes de autosimilitud, los índices de memoria, las dimensiones fraccionarias de las series de tiempo y las funciones de riesgo para los rendimientos son presentadas en la tabla 6: 
Tabla 6

Estimación de los exponentes de autosimilitud

\begin{tabular}{lccccccc}
\hline Paridad & EHG(1) & Mínimo & Máximo & $\boldsymbol{S}_{H}$ & $\boldsymbol{S}$ & $D$ & $\left(\boldsymbol{\aleph}_{k}\right)$ \\
\hline Dólar & 0.5141 & 0.5024 & 0.5268 & 0.0074 & 0.9148 & 1.4859 & 1.0852 \\
Euro & 0.5102 & 0.4926 & 0.5227 & 0.0099 & 0.9317 & 1.4898 & 1.0683 \\
Libra & 0.5201 & 0.5076 & 0.5270 & 0.0048 & 0.9275 & 1.4799 & 1.0725 \\
Yen & 0.5176 & 0.5062 & 0.5287 & 0.0070 & 0.9245 & 1.4824 & 1.0755 \\
\hline
\end{tabular}

Fuente: Elaboración propia con datos del Banco de México

La tabla 6 presenta los exponentes de autosimilitud, los rangos y la desviación estándar. Los índices de memoria $\mathbb{N}=\alpha H$ indican que los rendimientos presentan reversión a la media. Los índices $D=2-H$ indican que las dimensiones de las series de tiempo son fraccionarias. Las funciones de riesgo $=2-\aleph \mathfrak{i n d i c a n}$ que las medidas de riesgo están subestimadas porque ignoran las probabilidades de cambio en la tendencia de los rendimientos. Los resultados son congruentes con Climent Hernández et al. (2017), Climent Hernández y Aguilar Vázquez (2017) y Climent Hernández y Rodríguez Benavides (2018). Los resultados de las quince regresiones para estimar los exponentes de autosimilitud son mostrados en la tabla 7.

\section{Tabla 7}

ANOVA de los exponentes de autosimilitud

\begin{tabular}{|c|c|c|c|}
\hline Paridad & $R^{2}$ & $\mathrm{P}(T>t)$ & $\mathrm{P}(F>f)$ \\
\hline Dólar & {$[0.9937,0.9973]$} & [1.9E-20,1.6E-04] & [1.9E-20,1.6E-04] \\
\hline Euro & {$[0.9887,0.9969]$} & [5.4E-18,7.7E-05] & [5.4E-18,7.7E-05] \\
\hline Libra & {$[0.9949,0.9999]$} & [6.5E-21,4.1E-07] & [6.5E-21,4.1E-07] \\
\hline Yen & {$[0.9938,0.9964]$} & [1.4E-20,1.9E-04] & [1.4E-20,1.9E-04] \\
\hline
\end{tabular}

Fuente: Elaboración propia con datos del Banco de México

La Tabla 7 muestra los rangos de los coeficientes de determinación que indican que los exponentes de autosimilitud presentan una bondad de ajuste mayor que 98.87\%, los exponentes de autosimilitud son diferentes de cero, y el modelo es lineal, entonces, los exponentes de autosimilitud estimados con el método EHG(1) son no significativos y, por lo tanto, las paridades son autosimilares. La linealidad del método $\operatorname{EHG}(q)$, para los momentos $q=1,, 10$, 
determina si las paridades son autosimilares o multifraccionarias. Las estimaciones de los modelos son presentadas en la tabla 8 .

Tabla 8

Modelo de regresión lineal simple

\begin{tabular}{lllll}
\hline Paridad & EHG $(q)$ & $R^{2}$ & $\mathrm{P}(T>t)$ & $\mathrm{P}(F>f)$ \\
\hline Dólar & -0.0390 & 0.9726 & $1.55 \mathrm{E}-07$ & $1.55 \mathrm{E}-07$ \\
Euro & -0.0420 & 0.9765 & $8.36 \mathrm{E}-08$ & $8.36 \mathrm{E}-08$ \\
Libra & -0.0294 & 0.9753 & $1.02 \mathrm{E}-07$ & $1.02 \mathrm{E}-07$ \\
Yen & -0.0391 & 0.9781 & $6.39 \mathrm{E}-08$ & $6.39 \mathrm{E}-08$ \\
\hline
\end{tabular}

Fuente: Elaboración propia con datos del Banco de México

La tabla 8 presenta las pendientes. Los coeficientes de determinación indican que los exponentes de autosimilitud tienen una bondad de ajuste mayor que $97.26 \%$. Las pendientes son diferentes de cero, y el modelo es lineal, entonces, las paridades de las divisas son autosimilares. Por lo tanto, el análisis estadístico indica que los rendimientos de las paridades son autosimilares, y es pertinente aplicar procesos estocásticos $\alpha$-estables para la valuación de opciones sobre las paridades de los tipos de cambio del dólar, euro, libra esterlina y yen.

\section{Valuación de opciones europeas}

Las opciones son un derecho contingente que depende del precio subyacente en la fecha de vencimiento. La valuación de las opciones europeas a través del modelo gaussiano es:

$$
\begin{aligned}
& c\left(t, M_{t}\right)=M_{t} \exp (-r \tau) \Phi\left(d_{1}\right)-S \exp (-i \tau) \Phi\left(d_{2}\right) \\
& p\left(t, M_{t}\right)=S \exp (-i \tau) \Phi\left(-d_{2}\right)-M_{t} \exp (-r \tau) \Phi\left(-d_{1}\right)
\end{aligned}
$$

donde:

$$
d_{1}=\frac{\ln \left(\frac{M_{t}}{S}\right)+\left(i-r+\frac{\sigma^{2}}{2}\right) \tau}{\sigma \sqrt{\tau}} \text { y } d_{2}=d_{1}-\sigma \sqrt{\tau}
$$

Utilizando el supuesto de que los rendimientos presentan una distribución gaussiana donde $M_{t}$ es la paridad del tipo de cambio de las divisas, $S$ es el precio de liquidación, $i$ 
es la tasa de interés libre de riesgo nacional, $r$ es la tasa de interés libre de riesgo extranjera, $\sigma$ es la volatilidad de los rendimientos, y $\tau=T-t$ es el tiempo remanente donde $T$ es el periodo de vigencia de las opciones y $t$ es el periodo transcurrido. La valuación de las opciones europeas es realizada con del modelo $\alpha$-estable:

$$
\begin{aligned}
& c\left(t, M_{t}\right)=\left\{\begin{array}{l}
M_{t} \exp (-r \tau) \Phi(d ; \alpha, \beta)-S \exp (-i \tau) \Phi(d ; \alpha,-\beta) \\
M_{t} \exp (-r \tau)(1-\Phi(-d ; \alpha,-\beta))-S \exp (-i \tau)(1-\Phi(-d ; \alpha, \beta))
\end{array}\right. \\
& p\left(t, M_{t}\right)=\left\{\begin{array}{l}
S \exp (-i \tau) \Phi(-d ; \alpha, \beta)-M_{t} \exp (-r \tau) \Phi(-d ; \alpha,-\beta) \\
S \exp (-i \tau)(1-\Phi(d ; \alpha,-\beta))-M_{t} \exp (-r \tau)(1-\Phi(d ; \alpha, \beta))
\end{array}\right.
\end{aligned}
$$

donde:

$$
d=\frac{\ln \left(\frac{M_{t}}{S}\right)+\left(i-r-\beta \gamma^{\alpha} \sec (\theta)\right) \tau}{\gamma \tau^{\frac{1}{\alpha}}} \text { y } \theta=\frac{\alpha \pi}{2}
$$

El modelo $\alpha$-estable aplicado es similar al modelo de Climent Hernández y Cruz Matú (2017), donde $\alpha, \beta$ y $\gamma$ son los parámetros de las distribuciones $\alpha$-estables. La valuación de las opciones sobre divisas depende de factores exógenos como paridades, volatilidades, tasas de interés, y de factores endógenos como el precio de liquidación y el periodo de cobertura. Las paridades fueron obtenidas de banxico.org.mx. Las volatilidades estimadas son históricas. Las tasas de interés fueron obtenidas de banxico.org.mx, homefinance.nl y http:// es.global-rates.com. Los precios de liquidación fueron estimados como los precios forward, y el periodo de cobertura es del 2 de julio de 2018 con vencimiento el 26 de julio de 2018, es decir, 24 días naturales o 19 días hábiles. Los precios forward son:

$$
F_{t}=M_{t} \exp ((i-r) \tau)
$$

Los precios de las divisas en el momento de negociación y los precios de liquidación al vencimiento de las opciones sobre las paridades de los tipos de cambio son presentados en la tabla 9: 
Tabla 9

Precios de liquidación al vencimiento

\begin{tabular}{lll}
\hline Paridad & $M_{0}$ & $S$ \\
\hline Dólar & 19.6912 & 19.7625 \\
Euro & 22.9215 & 23.0401 \\
Libra & 25.8733 & 25.9918 \\
Yen & 0.1780 & 0.1789 \\
\hline
\end{tabular}

Fuente: Elaboración propia con datos del Banco de México y Home Finance

La Tabla 9 presenta los precios de las divisas en el momento de negociación de las opciones y los precios de liquidación al vencimiento para las opciones sobre las paridades que son calculadas con la ecuación . Los límites para la valuación de opciones europeas son:

$$
\begin{aligned}
& \text { máx }\left(M_{t} \exp (-r \tau)-S \exp (-i \tau), 0\right) \leq c\left(t, M_{t}\right) \leq M_{t} \\
& \text { máx }\left(S \exp (-i \tau)-M_{t} \exp (-r \tau), 0\right) \leq p\left(t, M_{t}\right) \leq S \exp (-i \tau)
\end{aligned}
$$

Los precios de las opciones satisfacen la ecuación (10) para evitar oportunidades de arbitraje. La valuación de las opciones europeas de compra gaussianas y $\alpha$-estables sobre las paridades de los tipos de cambio, para el periodo de vigencia, son presentadas en la figura 6 .

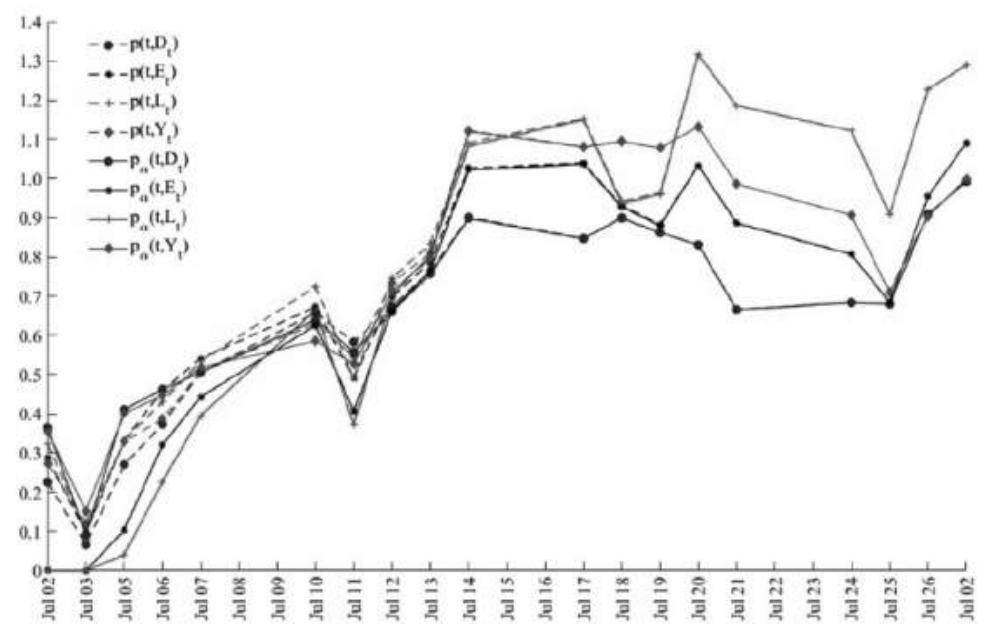

Figura 6. Valuación de opciones de compra Fuente: Elaboración propia con datos del Banco de México y Home Finance 
La figura 6 presenta las valuaciones de las opciones de compra gaussianas (líneas punteadas) y las valuaciones de las opciones de compra $\alpha$-estables (líneas continuas) sobre las paridades del dólar, euro, libra esterlina y yen². Las opciones de compra gasussianas sobre el euro y el libra esterlina tienen un valor superior que las opciones de compra $\alpha$-estables en el momento de emisión, y todas las opciones de compra están fuera de dinero en la fecha de vencimiento. La valuación de las opciones de venta gaussianas y $\alpha$-estables sobre las paridades, para el periodo de vigencia, es presentada en la figura 7.

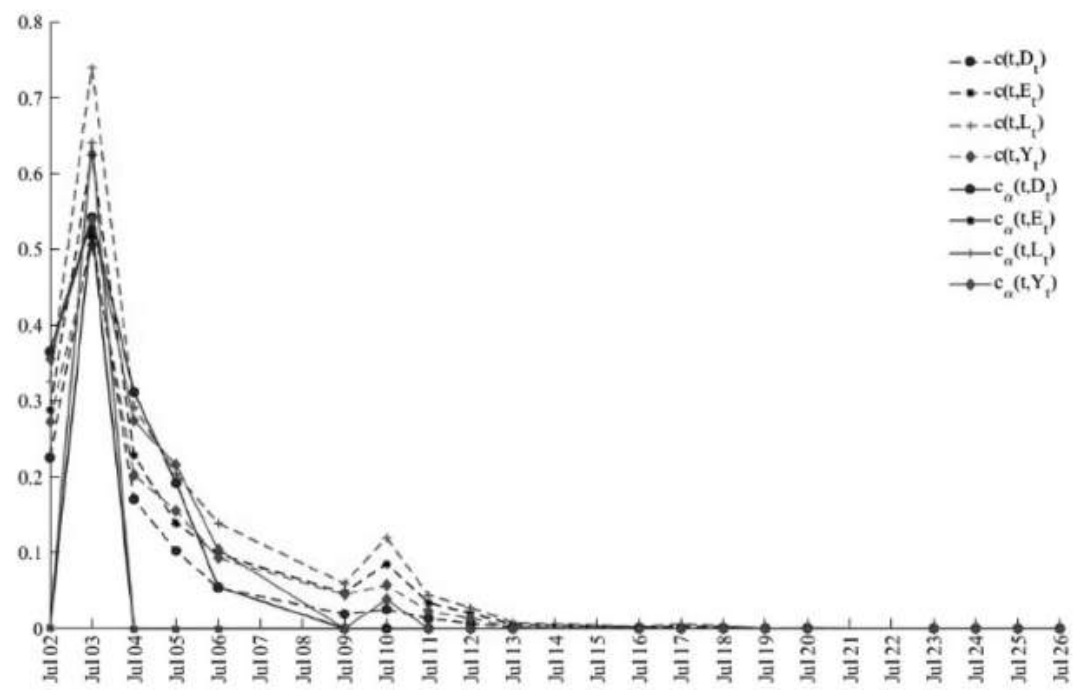

Figura 7.Valuación de opciones de venta

Fuente: Elaboración propia con datos del Banco de México y Home Finance

La figura 7 presenta las valuaciones de las opciones de venta gaussianas (líneas punteadas) y las valuaciones de las opciones de venta $\alpha$-estables (líneas continuas) sobre las paridades del tipo de cambio del dólar, euro, libra esterlina y yen ${ }^{3}$. Las opciones de venta gasussianas sobre el euro y la libra esterlina tienen un valor superior que las opciones de compra $\alpha$-estables en el momento de emisión, y todas las opciones de compra están dentro de dinero en la fecha de vencimiento.

Las figuras 6 y 7 indican que las opciones gaussianas sobre el dólar y sobre el yen son menos costosas que las opciones $\alpha$-estables, las opciones gaussianas sobre el euro y sobre la

\footnotetext{
${ }^{2}$ Las valuaciones de las opciones europeas de compra sobre el yen están multiplicadas por cien pesos.

${ }^{3}$ La valuación de opciones europeas de venta sobre el yen está multiplicada por cien pesos.
} 
libra esterlina más costosas que las opciones $\alpha$-estables, las opciones de compra están fuera de dinero en la fecha de vencimiento, y las opciones de venta están dentro de dinero en la fecha de vencimiento.

\section{Normas internacionales de información financiera}

Rodríguez Díaz (2017) indica que el Consejo de Normas Internacionales de Contabilidad (CNIC) o el International Accounting Standards Board (IASB) promulgó en julio de 2014 la norma de contabilidad de coberturas en un capítulo de la NIIF-9, sustituyendo a la NIC39 que regulaba la contabilidad de productos derivados y es de aplicación obligatoria a partir del 1 de enero de 2018.

Al negociar un producto derivado existen, desde un punto de vista contable, dos posibilidades:

1. Registrar el producto derivado como negociación o especulativo a valor razonable.

2. Registrar el producto derivado como producto de cobertura.

La NIIF-9 alinea los objetivos de gestión de riesgos con la contabilidad simplificando los requisitos de la eficiencia de cobertura introduciendo obligaciones de información sobre las actividades de gestión de riesgos para que la contabilidad de coberturas transmita el objetivo y el efecto de los productos de cobertura (derivados) con carácter opcional. Para la NIC39, los productos de cobertura son los productos derivados, con la excepción de que son utilizados productos financieros no derivados para cubrir riesgos de tipo de cambio y la NIIF-9 no rige el cumplimiento de la contabilidad de coberturas cuando el producto es o no un derivado. Las diferencias principales con respecto a la NIC39 son:

1. Las exposiciones globales que incluyen un derivado con circunstancias concretas en las que son cubren posiciones netas son posibles de asignar en la NIIF-9 y la NIC39 lo prohibía.

2. La contabilización del valor extrínseco de las opciones en las relaciones de cobertura, de flujos de efectivo o de valor razonable, cuya variación de valor razonable es posible diferir bajo ciertas reglas como un costo por la cobertura es modificado en la NIIF-9 y en la NIC39 se llevaba este componente a resultados como una ineficiencia.

3. La evaluación de la eficiencia está alineada con la gestión de riesgo con el principio de relación económica en la NIIF-9 y en la NIC39 con la regla cuantitativa de cobertura prospectiva y retrospectiva de 80 a $125 \%$. La evaluación retrospectiva deja de ser un requisito, pero es utilizado para registrar la ineficiencia. 
4. La posibilidad de valor razonable es una solución para casos en los que es imposible aplicar la contabilidad de coberturas.

En la NIC39 al registrar el producto derivado como producto de cobertura son aplicadas normas especiales de contabilidad de coberturas para minimizar asimetrías contables que surgen porque el producto derivado está compensando otra posición que no es valorada a valor razonable con cambios en el estado de resultados o que no aparece en balance.

En la NIIF-9, el valor extrínseco es diferido como un costo por la cobertura, entonces, la cobertura con opciones es atractiva nuevamente. El modelo de contabilidad de coberturas de la NIC39 es complejo, estricto y no refleja la realidad económica de las políticas de gestión de riesgos en el contexto del modelo de negocio. La NIIF-9 alinea los objetivos de gestión de riesgos con la contabilidad para reflejar las actividades de gestión de riesgos transmitiendo el objetivo y el efecto de los productos de cobertura con carácter opcional. La NIIF-9 para productos derivados indica los requerimientos para clasificar y medir productos financieros, y la contabilidad de cobertura. El costo de los productos derivados es clasificado en el balance general como un activo o un pasivo, dependiendo de los derechos u obligaciones establecidas en el contrato. El estado de resultados presenta los cambios periódicos de las valuaciones de los activos o pasivos financieros.

En México, la contabilidad está regida bajo la norma de información financiera (NIF C2) que establece que los productos derivados que constituyen una inversión temporal, con un plazo menor a un año, que cotizan en el mercado de valores, y los productos de inversión o de cobertura son clasificados como inversiones temporales y son presentados en los activos circulantes o en los pasivos de corto plazo según corresponda a los derechos y obligaciones.

\section{Estado de resultados}

Los cambios en el valor del subyacente generan cambios en el valor de las opciones. Por lo tanto, la posición corta registra como ganancias las primas cobradas y como pérdidas los pagos contingentes, y la posición larga registra como ganancias los pagos contingentes y como pérdidas las primas pagadas.

\section{Balance general}

Las opciones son presentadas en el activo o en el pasivo, respectivamente, las opciones con la posición larga, que otorgan el derecho, pero no la obligación, son presentadas en el activo, en bancos como la prima pagada, y las opciones con la posición corta, que otorgan 
la obligación, son presentadas en el activo, en bancos como la prima cobrada y en el pasivo como el pago contingente.

Contabilidad de opciones europeas de compra y venta

Suponiendo las opciones amparan diez mil divisas, entonces, la emisión o adquisición para la cobertura es por cien lotes que amparan cien divisas cada uno. Las cuentas $T$ de la posición larga de las opciones de compra y de venta sobre las divisas con presentadas en la tabla 10.

Tabla 10

Cuentas $T$ de la posición larga de las opciones de compra y de venta

Productos derivados

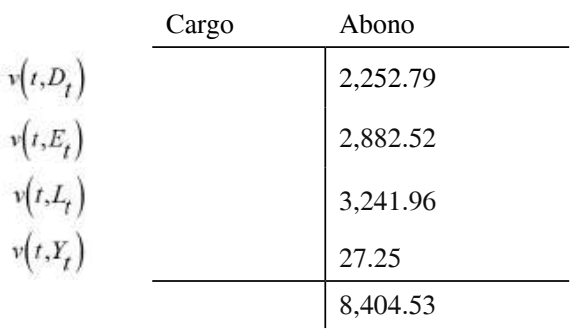

Productos derivados

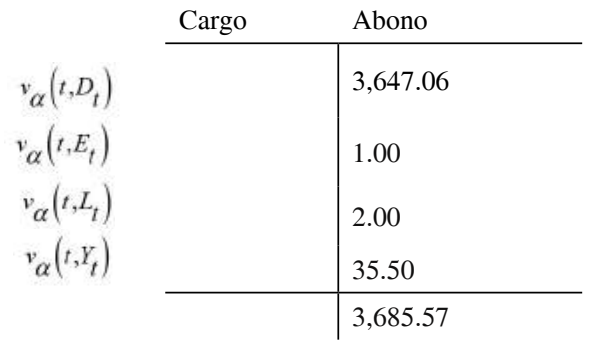

Bancos

\begin{tabular}{l|l} 
Cargo & \multicolumn{1}{c}{ Abono } \\
\hline $2,252.79$ & \\
$2,882.52$ & \\
$3,241.96$ & \\
27.25 & \\
\hline $8,404.53$ &
\end{tabular}

Bancos

\begin{tabular}{l|l} 
Cargo & Abono \\
\hline $3,647.06$ & \\
1.00 & \\
2.00 & \\
35.50 & \\
\hline $3,685.57$ &
\end{tabular}

Fuente: Elaboración propia

La tabla 10 presenta las cuentas $T$ para la posición larga de las opciones de compra y de venta sobre las divisas. Las primas pagadas ascienden a $\$ 8,404.53$ y $\$ 3,608.57$, respectivamente. Las opciones gaussianas sobre el dólar estadounidense y sobre el yen son menos costosas que las opciones $\alpha$-estables. Las opciones gaussianas sobre el euro y sobre 
la libra esterlina más costosas que las opciones $\alpha$-estables. Las opciones de compra están fuera de dinero en la fecha de vencimiento y las opciones de venta están dentro de dinero en la fecha de vencimiento, entonces, los poseedores gaussianos están sobreestimando el valor del portafolio de opciones de compra o de venta, y la prima por la cobertura es mayor que la de los poseedores $\alpha$-estables. Los poseedores adquieren derechos con los emisores, es decir, adquieren ganancias contingentes. Las cuentas $T$ de la posición corta de las opciones de compra y de venta sobre las divisas son presentadas en la tabla 11.

Tabla 11

Cuentas $T$ de la posición corta de las opciones de compra o de venta

\begin{tabular}{ll|l} 
& \multicolumn{2}{c}{ Productos derivados } \\
\cline { 3 - 3 }$v\left(t, D_{t}\right)$ & $2,252.79$ & \\
$v\left(t, E_{t}\right)$ & $2,882.52$ & \\
$v\left(t, L_{t}\right)$ & $3,241.96$ & \\
$v\left(t, Y_{t}\right)$ & 27.25 & \\
\cline { 2 - 3 } & $8,404.53$ &
\end{tabular}

\begin{tabular}{|c|c|}
\hline \multirow{6}{*}{ Cargo } & Bancos \\
\hline & Abono \\
\hline & $2,252.79$ \\
\hline & $2,882.52$ \\
\hline & $3,241.96$ \\
\hline & 27.25 \\
\hline & $8,404.53$ \\
\hline
\end{tabular}

Productos derivados

\begin{tabular}{l|l}
\multicolumn{1}{c}{ Bancos } \\
Cargo & $\begin{array}{l}\text { Abono } \\
1.00 \\
2.047 .06 \\
\\
\end{array}$ \\
\hline
\end{tabular}

Fuente: Elaboración propia

La tabla 11 presenta las cuentas $T$ para la posición corta de las opciones de compra y de venta sobre las divisas y se observa un comportamiento similar a la posición larga, pero los emisores de las opciones europeas de compra y de venta adquieren obligaciones con los poseedores, es decir, adquieren pérdidas contingentes. 
Las variaciones en el valor de las opciones son registradas en el estado de resultados, de acuerdo con la (NIF B3), el resultado integral de financiamiento (RIF) está conformado por ingresos y gastos relacionados con actividades financieras. El RIF presenta el valor de activos o pasivos financieros, ganancias o pérdidas por las valuaciones. El resultado integral del financiamiento de la posición larga de las opciones europeas de compra como parte del estado de resultados al 31 de julio de 2018 es presentado en la tabla 12.

Tabla 12

Estado de resultados de la posición larga de opciones de compra al 31 de julio de 2018

\begin{tabular}{lll}
\multicolumn{3}{c}{ Resultado del financiamiento } \\
$\Pi_{c\left(t, M_{t}\right)}$ Ganancia por cobertura & 0.00 \\
$\Pi_{c\left(t, M_{t}\right)}$ & Pérdida por cobertura & $-8,404.53$ \\
& Resultado del financiamiento & \\
$\Pi_{c}\left(t, M_{t}\right)$ & Ganancia por cobertura & 0.00 \\
$\Pi_{c}\left(t, M_{t}\right)$ & Pérdida por cobertura & $3,685.57$ \\
${ }$ & & $-3,685.57$
\end{tabular}

Fuente: Elaboración propia

La Tabla 12 presenta el resultado del financiamiento de la posición larga de las opciones europeas de compra como parte del estado de resultados al 31 de julio de 2018. La pérdida de la cobertura con el portafolio gaussiano $(\$ 8,404.53)$ es mayor que la pérdida del portafolio $\alpha$-estable $(\$ 3,685.57)$ y la diferencia entre la ganancia por la cobertura y la prima pagada es reportada en bancos del balance general. El resultado del financiamiento de la posición larga de las opciones europeas de venta como parte del estado de resultados al 31 de julio de 2018 es presentada en la tabla 13. 
Tabla 13

Estado de resultados de la posición larga de opciones de venta al 31 de julio de 2018

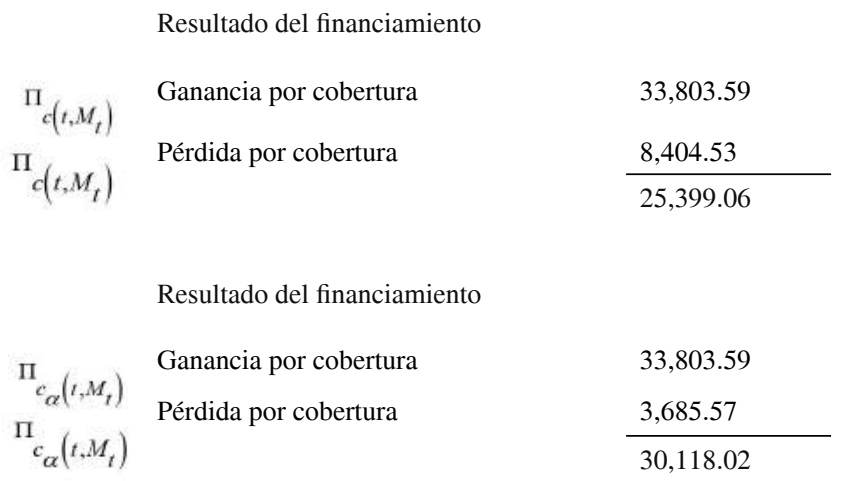

Fuente: Elaboración propia

La tabla 13 presenta el resultado del financiamiento de la posición larga de las opciones europeas de venta como parte del estado de resultados al 31 de julio de 2018. La ganancia neta de la cobertura con el portafolio gaussiano $(\$ 25,399.06)$ es menor que la ganancia neta de la cobertura con el portafolio $\alpha$-estable $(\$ 30,118.02)$. Por lo tanto, la ganancia neta del portafolio gaussiano es menor que la ganancia neta del portafolio $\alpha$-estable y la diferencia entre la ganancia por la cobertura y la prima pagada es reportada en el rubro bancos del balance general. El resultado integral del financiamiento de la posición corta de las opciones europeas de compra como parte del estado de resultados al 31 de julio de 2018 es presentado en la tabla 14.

Tabla 14

Estado de resultados de la posición corta de opciones de compra al 31 de julio de 2018

\begin{tabular}{lll}
\multicolumn{3}{c}{ Resultado del financiamiento } \\
$\Pi_{c\left(t, M_{t}\right)}$ Ganancia por cobertura & $8,404.53$ \\
$\Pi_{c\left(t, M_{t}\right)}$ & Pérdida por cobertura & 0.00 \\
& Resultado del financiamiento & $8,404.53$ \\
$\Pi_{c}\left(t, M_{t}\right)$ & Ganancia por cobertura & \\
$\Pi_{c}\left(t, M_{t}\right)$ & Pérdida por cobertura & $3,685.57$ \\
${ }$ &
\end{tabular}

Fuente: Elaboración propia 
La Tabla 14 presenta el resultado del financiamiento de la posición corta de las opciones europeas de compra como parte de los resultados al 31 de julio de 2018. La ganancia por emitir la cobertura con el portafolio gaussiano es mayor que la ganancia por emitir la cobertura con el portafolio $\alpha$-estable. La ganancia del portafolio gaussiano es mayor que la ganancia del portafolio $\alpha$-estable y la diferencia entre la ganancia y la pérdida de la cobertura es reportada en el rubro bancos del balance general. El resultado integral del financiamiento de la posición corta de las opciones europeas de venta como parte del estado de resultados al 31 de julio de 2018 es presentado en la tabla 15.

Tabla 15

Estado de resultados de la posición corta de opciones de venta al 31 de julio de 2018

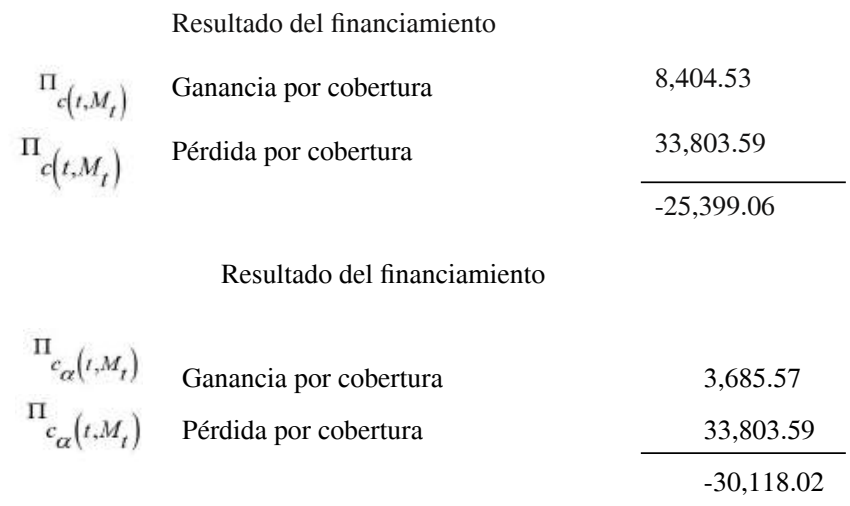

Fuente: Elaboración propia

La tabla 15 presenta el resultado del financiamiento de la posición corta de las opciones europeas de venta como parte del estado de resultados al 31 de julio de 2018. La pérdida neta por la emisión de la cobertura con el portafolio gaussiano $(\$ 25,399.06)$ es menor que la pérdida neta por la emisión de la cobertura con el portafolio $\alpha$-estable $(\$ 30,118.02)$. Por lo tanto, la pérdida neta del portafolio gaussiano es menor que la pérdida neta del portafolio $\alpha$-estable y la diferencia entre la ganancia por la cobertura y la pérdida por la cobertura es reportada en el rubro bancos del balance general. Entonces, la pérdida del portafolio gaussiano es menor que la pérdida del portafolio $\alpha$-estable.

La NIIF-9 y la NIF C2 indican que se presenta el registro contable con el valor de las opciones en el balance general. Las pérdidas de la posición larga de las opciones de compra como parte del balance general son presentadas en la tabla 16. 
Tabla 16

Balance general de la posición larga de opciones de compra al 31 de julio de 2018

$\begin{array}{llr}\Pi_{c\left(t, M_{t}\right)} & \text { Bancos } & -8,404.53 \\ & \text { Productos derivados } & -8,404.53\end{array}$

Bancos $\quad-3,685.57$

$\Pi_{c_{\alpha}\left(t, M_{t}\right)} \quad$ Productos derivados $\quad-3,685.57$

Fuente: Elaboración propia

La tabla 16 presenta el total de las primas pagadas por las opciones de compra sobre las divisas como un egreso por la cobertura. Las ganancias de la posición larga de las opciones de venta como parte del balance general son presentadas en la tabla 17.

Tabla 17

Balance general de la posición larga de opciones de venta al 31 de julio de 2018

$\begin{array}{clc} & \text { Bancos } & 25,399.06 \\ \Pi_{p\left(t, M_{t}\right)} & \text { Productos derivados } & 25,399.06 \\ & & \\ & \text { Bancos } & 30,118.02 \\ \Pi_{p_{\alpha}\left(t, M_{t}\right)} & \text { Productos derivados } & 30,118.02\end{array}$

Fuente: Elaboración propia

La tabla 17 presenta la diferencia entre el total de las ganancias de los pagos de liquidación al vencimiento de las opciones de venta y el total de las primas pagadas por las opciones de venta sobre las divisas como un ingreso porque el pago de liquidación al vencimiento es mayor que las primas pagadas. Las ganancias de la posición corta de las opciones de compra como parte del balance general son presentadas en la tabla 18. 
Tabla 18

Balance general de la posición corta de opciones de compra al 31 de julio de 2018

$\begin{array}{lll}\Pi_{c\left(t, M_{t}\right)} & \text { Bancos } & 8,404.53 \\ & \text { Productos derivados } & 8,404.53 \\ & & 3,685.57 \\ \Pi_{c{ }_{\alpha}\left(t, M_{t}\right)} & \text { Bancos } & 3,685.57\end{array}$

Fuente: Elaboración propia

La tabla 18 presenta el total de las primas cobradas por las opciones de compra sobre las divisas como un ingreso por la cobertura otorgada. Las pérdidas de la posición corta de las opciones de venta como parte del balance general son presentadas en la tabla 19.

Tabla 19

Balance general de la posición corta de opciones de venta al 31 de julio de 2018

$\begin{array}{clr}\Pi_{p\left(t, M_{t}\right)} & \text { Bancos } & -25,399.06 \\ & \text { Productos derivados } & -25,399.06 \\ & \text { Bancos } & -30,118.02 \\ \Pi_{p_{\alpha}\left(t, M_{t}\right)} & \text { Productos derivados } & -30,118.02\end{array}$

Fuente: Elaboración propia

La Tabla 19 presenta la diferencia entre el total de las primas cobradas por las opciones de venta sobre las divisas y el total de las pérdidas de los pagos de liquidación al vencimiento de las opciones de venta como un egreso porque el pago de liquidación al vencimiento es mayor que el total de las primas cobradas.

Suponiendo que la emisión de las opciones es por diez mil divisas y sólo se tiene una posición individual, es decir, se tienen o se emiten cien lotes de opciones sobre sólo una de las divisas, entonces, la posición larga y la posición corta sobre opciones europeas de compra y de venta presenta los resultados siguientes:

La posición larga de las opciones de compra gaussianas sobre el dólar y sobre el yen presenta pérdidas menores que las opciones de compra $\alpha$-estables y las opciones de compra 
gaussianas sobre el euro y sobre la libra esterlina y presentan pérdidas mayores que las opciones de compra $\alpha$-estables.

La posición larga de las opciones de venta gaussianas sobre el dólar y sobre el yen presenta ganancias mayores que las opciones de venta $\alpha$-estables y las opciones de venta gaussianas sobre el euro y sobre la libra esterlina y presentan ganancias menores que las opciones de compra $\alpha$-estables.

La posición corta de las opciones de compra gaussianas sobre el dólar y sobre el yen presenta ganancias menores que las opciones de compra $\alpha$-estables y las opciones de compra gaussianas sobre el euro y sobre la libra esterlina y presentan ganancias mayores que las opciones de compra $\alpha$-estables.

La posición corta de las opciones de venta gaussianas sobre el dólar y sobre el yen presenta pérdidas mayores que las opciones de venta $\alpha$-estables y las opciones de venta gaussianas sobre el euro y sobre la libra esterlina y presentan pérdidas menores que las opciones de compra $\alpha$-estables.

Los portafolios de opciones, con las posiciones largas y cortas, que fueron analizados son estrategias para coberturas y combinaciones (opciones europeas de compra y venta de la misma serie):

1. Cono posición larga (long straddle). Los inversionistas esperan cambios relevantes en los precios subyacentes y crean el portafolio siguiente:

1.1 La posición larga de un portafolio de opciones europeas de compra.

1.2 La posición larga de un portafolio de opciones europeas de venta de la misma serie.

El cono largo es conocido también como montura larga (bottom straddle) y genera pérdidas acotadas si el portafolio de subyacentes tiene precios cercanos a los precios de liquidación, genera ganancias limitadas si el portafolio de subyacentes tiene precios menores que los precios de liquidación, y genera ganancias ilimitadas si el portafolio de subyacentes tiene precios mayores que los precios de liquidación, es decir, los inversionistas esperan que los precios subyacentes sean lejanos de los precios de liquidación.

2. Cono posición corta (short straddle). Los inversionistas esperan cambios no significativos en los precios subyacentes y crean el portafolio siguiente:

2.1 La posición corta de un portafolio de opciones europeas de compra.

2.2 La posición corta de un portafolio de opciones europeas de venta de la misma serie.

El cono corto es conocido también como montura corta (top straddle) y genera ganancias acotadas si el portafolio tiene precios cercanos a los precios de liquidación, genera pérdidas limitadas si el portafolio tiene precios menores que los precios de liquidación, y genera pérdidas ilimitadas si el portafolio de subyacentes tiene precios mayores que los precios de 
liquidación, es decir, los inversionistas esperan que los precios subyacentes sean cercanos a los precios de liquidación.

Las combinaciones de cobertura que es posible analizar también son las tiras largas (long strips), tiras cortas (short strips), correas largas (long straps), y correas cortas (short straps). Así como otras coberturas y otras estrategias con portafolios de opciones, y analizar periodos más extensos de cobertura para aplicar la contabilidad de conformidad con la NIIF-9.

Las recomendaciones para los inversionistas son realizar los análisis para identificar, cuantificar y minimizar los riesgos. Realizar coberturas dinámicas con el producto financiero objeto de cobertura (divisa). Crear estrategias con portafolios que acotan las pérdidas potenciales adquiridas por las obligaciones contraídas que es equivalente al reaseguro (transfiriendo el riesgo con la negociación adecuada de otro producto, incluyendo derivados).

\section{Conclusiones}

Los análisis realizados a rendimientos presentan acontecimientos estilizados. Los estadísticos descriptivos y los parámetros $\alpha$-estables indican que los inversionistas obtienen en promedio utilidades positivas. El mayor riesgo medido con la desviación estándar o con el parámetro de escala es para el yen, el euro, la libra esterlina y el dólar, es decir, el dólar es el la divisa con mayor rendimiento y con menor riesgo, el euro es la divisa con el segundo mejor rendimiento y es el tercer tipo de cambio más riesgoso, la libra esterlina es la divisa con el tercer mejor rendimiento y es el segundo tipo de cambio más riesgoso, el yen es cuarto mejor rendimiento y el tipo de cambio más riesgoso. Los rendimientos presentan coeficientes de asimetría positivos, entonces, los rendimientos promedio son mayores que la mediana y la moda y las distribuciones se extienden más hacia las ganancias que hacia las pérdidas. Los rendimientos presentan coeficientes de curtosis positivos, entonces, las distribuciones gaussianas subestiman las utilidades relevantes y nulas, pero sobrestiman las utilidades moderadas.

Las estimaciones de los parámetros $\alpha$-estables son consistentes con los estadísticos descriptivos porque indican la presencia de asimetría y leptocurtosis, y las estimaciones de los intervalos de confianza de los parámetros de las distribuciones $\alpha$-estables confirman que los rendimientos presentan leptocurtósis y asimetría positiva, entonces, las distribuciones $\alpha$ -estables cuantifican más apropiadamente las utilidades y los riesgos que las distribuciones gaussianas, hipótesis que son comprobadas con las pruebas de bondad de ajuste. Los niveles de significación descriptivos confirman que las distribuciones $\alpha$-estables son eficientes y estadísticamente no significativas para modelar el comportamiento empírico de los rendimientos estudiados. 
Las estimaciones de los exponentes de autosimilitud indican que los rendimientos presentan reversión a la media. Las dimensiones de las series de tiempo son fraccionarias, entonces, las medidas de riesgo como el parámetro de escala, el valor en riesgo (VaR) o el valor en riesgo condicional (CVaR) están subestimadas por sí mismas porque ignoran las probabilidades de cambio de tendencia de los rendimientos indicadas por los exponentes de autosimilitud y que son corregidas con las funciones de riesgo. Las dimensiones de las series de tiempo son mayores que la unidad y los riesgos son mayores porque la serie de tiempo ocupa un área mayor en el plano que una curva determinista. Los niveles de significación descriptivos indican que el modelo es lineal y que los rendimientos son autosimilares. Por lo tanto, los procesos estocásticos $\alpha$-estables son idóneos y superan a los procesos estocásticos de Wiener para la valuación de opciones sobre los tipos de cambio estudiados.

La valuación de las opciones indica que las opciones de compra gaussianas sobre el dólar $\mathrm{y}$ el yen presentan un valor de negociación menor que las opciones $\alpha$-estables porque en el momento de negociación $\Phi(d, \alpha, \beta)-\Phi(d ; \alpha,-\beta)>\Phi\left(d_{1}\right)-\Phi\left(d_{2}\right)$, por lo tanto, las valuaciones gaussianas son menores que las $\alpha$-estables. Las opciones de compra gaussianas sobre el euro y la libra esterlina presentan un valor de negociación mayor que las opciones $\alpha$-estables porque en el momento de negociación $\Phi(d, \alpha, \beta)-\Phi(d ; \alpha,-\beta)<\Phi\left(d_{1}\right)-\Phi\left(d_{2}\right)$, por lo tanto, las valuaciones gaussianas son mayores que las $\alpha$-estables. Las opciones de venta gaussianas sobre el dólar y el yen presentan un valor de negociación menor que las opciones $\alpha$-estables porque en el momento de negociación $\Phi(d, \alpha, \beta)-\Phi(d ; \alpha,-\beta)>\Phi\left(d_{1}\right)-\Phi\left(d_{2}\right)$, por lo tanto, las valuaciones gaussianas son menores que las $\alpha$-estables. Las opciones de venta gaussianas sobre el euro y la libra esterlina presentan un valor de mayor que las opciones $\alpha$-estables porque en el momento de negociación se tiene que $\Phi(d, \alpha, \beta)-\Phi(d ; \alpha,-\beta)<\Phi\left(d_{1}\right)-\Phi\left(d_{2}\right)$, por lo tanto, las valuaciones gaussianas son mayores que las $\alpha$-estables.

La cobertura del portafolio $\alpha$-estable es menor que la cobertura por el portafolio gaussiano, los seguros financieros con opciones $\alpha$-estables de compra sobre el dólar y el yen son más costosos que los seguros con opciones gaussianas, y los seguros con opciones $\alpha$-estables de compra sobre el euro y la libra esterlina son menos costosos que los seguros con opciones gaussianas. Por lo tanto, las opciones $\alpha$-estables sobre el euro y la libra esterlina cuantificaron el riesgo de tipo de cambio adecuadamente porque estuvieron fuera de dinero y los costos por las coberturas de los riesgos de los tipos de cambio fueron menores y más apegados a las distribuciones empíricas. Las opciones $\alpha$-estables sobre el dólar, las opciones $\alpha$-estables sobre el yen cuantificaron el riesgo de tipo de cambio adecuadamente. Por lo tanto, las opciones $\alpha$ -estables sobre el dólar y el yen son más riesgosas para la posición larga. 
Las pérdidas con la posición corta del portafolio $\alpha$-estable de venta son mayores que las pérdidas del portafolio gaussiano. Las pérdidas por la emisión de las opciones $\alpha$-estables de venta sobre el dólar y el yen son menores que las pérdidas por las opciones gaussianas, y las pérdidas por las opciones $\alpha$-estables de compra sobre el euro y la libra esterlina son mayores que las ganancias por las opciones gaussianas. Las coberturas emitidas con opciones $\alpha$-estables de venta sobre el euro y la libra esterlina son las que generan que las pérdidas por las coberturas $\alpha$-estables de venta sean mayores que las coberturas con opciones gaussianas de venta, pero si las coberturas con opciones $\alpha$-estables de venta sobre el euro y la libra esterlina cambian los precios de liquidación, entonces, las pérdidas máximas para las posiciones cortas están presentes cuando los precios de liquidación son $S=23.62$ y $S=26.65$, pero si los precios de liquidación son $S \leq 21.9498$ y $S \leq 24.7034$, entonces, las pérdidas por las coberturas $\alpha$ -estables de venta son nulas, además, si las opciones de venta sobre el euro y la libra esterlina están fuera de dinero en la fecha de negociación, las pérdidas respectivas son crecientes hasta que $S=23.62$ y $S=26.65$, y son decrecientes y asintóticas a las pérdidas gaussianas cuando los precios de liquidación respectivos son $S>23.62$ y $S>26.65$. El análisis de sensibilidad en función de los precios de liquidación es importante para las posiciones cortas porque permite conocer las utilidades en función de los precios de liquidación y el valor de las opciones en el instante de negociación. El análisis de sensibilidad para los precios de liquidación en las opciones sobre el euro y la libra esterlina, indica que las pérdidas gaussianas son mayores que las pérdidas $\alpha$-estables cuando los precios de liquidación son 22.50 y 25.00. Por lo tanto, la recomendación es que los inversionistas con la posición corta utilicen el reaseguro con productos financieros (divisas, opciones, o contratos a plazo) como cobertura para minimizar las pérdidas por las obligaciones adquiridas. Los inversionistas con posición corta tienen que identificar los riesgos, cuantificarlos adecuadamente y minimizarlos para cubrir parcialmente las obligaciones contraídas y minimizar las pérdidas potenciales.

Las estrategias con coberturas y combinaciones también son recomendaciones para acotar las pérdidas potenciales por las obligaciones contraídas al acotar las ganancias potenciales creando portafolios categorizados como opciones sintéticas de compra o venta, diferenciales de compra o venta al alza o a la baja, diferenciales mariposa, conos, tiras o corras con posiciones largas o cortas de acuerdo a las tendencias de los precios subyacentes.

Las opciones sobre las paridades de los tipos de cambio son europeas y negociadas a valor razonable, es decir, sólo son ejercidas en la fecha de vencimiento, pero los inversionistas pueden negociar (comprar o vender) en mercados primarios, o secundarios, entonces, los inversionistas no tienen la obligación de esperar hasta la fecha de vencimiento. Por lo tanto, los portafolios son dinámicos de acuerdo con las necesidades de cobertura. 
El análisis y la aplicación de ajustes no significativos son importantes para que la valuación de opciones cuantifique adecuadamente el riesgo de mercado como lo hacen los seguros, entonces, los mercados esperan que actuarios, economistas e ingenieros financieros identifiquen, minimicen y cuantifiquen adecuadamente los riesgos con ajustes no significativos y que los contadores apliquen adecuadamente la NIIF.

\section{Referencias}

Bachelier, L. J. B. A. (1900). Théorie de la spéculation, PhD thesis, Sorbonne, Annales scientifiques de l'École Normale Supérieure. https://doi.org/10.24033/asens.476.

Barone Adesi, G. y Whaley, R. (1987). Efficient analytic approximation of american options values, The Journal of Finace 42(2): 301-320. https://doi.org/10.1111/j.1540-6261.1987.tb02569.x.

Barunik, J. y Kristoufek, L. (2010). On Hurst exponent estimation under heavy-tailed distributions, Physica A: Statistical Mechanics and its Applications, 389: 3844-3855. https://doi.org/10.1016/j. physa.2010.05.025.

Belov, I., Kabašinskas, A. y Sakalauskas, L. (2006). A study of stable models of stock markets, Information Technology and Control, 35(1):34-56.

Black, F. y Scholes, M. (1973). The pricing of options and corporate liabilities, Journal of Political Economy, 81:637-654. https://doi.org/10.1086/260062.

Boness, J. (1964). Elements of a theory of stock-option values, Journal of Political Economy, 72(2): 163-175. https://doi.org/10.1086/258885.

Brennan, M. J. y Schwartz, E. S. (1977). The valuation of american put options, The Journal of Finance 32(2): 449-462. https://doi.org/10.1111/j.1540-6261.1977.tb03284.x.

Čížek, P., Härdle, W. y Weron, R. (2005). Stable Distributions. Statistical Tools for Finance and Insurance. Berlin, SPRINGER: 21-44. https://doi.org/10.1007/b139025.

Carr, P. y Wu, L. (2003). The finite moment log stable process and option pricing, The Journal of Finance LVIII(2): 753-778. https://doi.org/10.1111/1540-6261.00544.

Climent Hernández, J. A. (2014). La ecuación de segundo grado en la estimación de parámetros de la martingala y la valuación de opciones americanas a través de la programación dinámica estocástica. Estocástica: Finanzas y Riesgo, 4(2): 155-190.

Climent Hernández, J. A. y Aguilar Vázquez, A. (2017). Medidas de riesgo de un índice bursátil de la zona euro, XXII Congreso Internacional de Contaduría Administración e Informática, Premio de Investigación Arturo Díaz Alonso 2017 en el área de finanzas. Consultado el 1 de marzo de 2019. http://premio.investiga.fca.unam.mx/docs/XXII/6.pdf.

Climent Hernández, J. A. y Cruz Matú, C. (2017). Pricing of a structured product on the SX5E when the uncertainty of returns is modeled as a log-stable process, Contaduría y Administración, 62(4): 1160-1182. http://dx.doi.org/10.1016/j.cya.2017.06.012.

Climent Hernández, J. A., Hoyos Reyes, L. F. y Rodríguez Benavides, D. (2017). The $\alpha$-stable processes and their relationship with the exponent of self-similarity: Exchange rates of USA Dollar, Canadian Dollar, Euro and Yen, Contaduría y Administración, 62(5): 1501-1522. https://doi.org/10.1016/j.cya.2017.10.002.

Climent Hernández, J. A. y Rodríguez Benavides, D. (2018). Superficie de riesgo y mapeo de los índices de riesgo del IPC, IBEX35 y S\&P500, Capítulo 13 de Economía Financiera: Teoría, Modelos e Investigación Aplicada, 351-380, Universidad Autónoma Metropolitana Iztapalapa, ISBN: 978607-28-1688-6. Publicación próxima. 
Climent Hernández, J. A. y Venegas Martínez, F. (2013). Valuación de opciones sobre subyacentes con rendimientos $\alpha$-estables, Contaduría y Administración, 58(4): 119-150. http://dx.doi.org/10.1016/S0186-1042(13)71236-1.

Contreras Piedragil, C. E. y Venegas Martínez, F. (2011). Valuación de opciones sobre activos subyacentes con distribuciones estables. Estocástica, 1(1): 55-71.

Cox, J. C. y Ross, S. A. (1976). The valuation of options for alternative stochastic processes, Journal of Financial Economics 3(1): 145-166. https://doi.org/10.1016/0304-405X(76)90023-4.

Cox, J. C., Ross, S. A. y Rubinstein, M. (1979). Option pricing: A simplified approach, Journal of Financial Economics, 7(1): 229-263. https://doi.org/10.1016/0304-405X(79)90015-1.

Dostoglou, S. y Rachev, S. T. (1999). Stable distributions and term structure of interest rates, Mathematical and Computer Modelling, 29(10): 57-60. https://doi.org/10.1016/S0895-7177(99)00092-8.

Esscher, F. (1932). On the probability function in the collective theory of risk, Scandinavian Actuarial Journal 17: 175-195. https://doi.org/10.1080/03461238.1932.10405883.

Fama, E. (1963). Mandelbrot and the stable paretian hypothesis, Journal of Business, 36(4): 420-429. https://doi. org/10.1086/294633.

Fama, E. (1965a). Portfolio analysis in a stable paretian market, Management Science, 11(3): 404-419. https://doi. org/10.1287/mnsc.11.3.404.

Fama, E. (1965b). The behavior of stock market prices, Journal of Business, 38(1): 34-105. https://doi.org/10.1287/ mnsc.11.3.404.

Girsanov, I. V. (1960). On transforming a certain class of stochastic processes by absolutely continuous substitution of measures, Theory of Probability and its Applications 5: 285-301. https://doi.org/10.1137/1105027.

Glover, H. y Werner, E. M. (2015). Teaching IFRS: Options for instructors, Advances in Accounting Education: Teaching and Curriculum Innovations, 16: 113-131.

https://doi.org/10.1108/S1085-462220150000016006.

Gómez Pinto, I., Mapén Franco, de J. F. y Climent Hernández, J. A. (2019). Valuación y contabilización de opciones europeas: compra y venta considerando la paridad del tipo de cambio del dólar, euro, libra y yen, Journal of Research in Business and Management, 7(2): 14-23.

Heston, S. L. (1993). A closed-form solution for options with stochastic volatility, The Review of Financial Studies 6(2): 327-343.

Hull, J. C. y White, A. (1987). The pricing of options on assets with stochastic volatilities, Journal of Finance 42(2): 281-300. https://doi.org/10.1111/j.1540-6261.1987.tb02568.x.

Hurst, S. R., Platen, E. y Rachev, S. T. (1999). Option pricing for a logstable asset price model, Mathematical and Computer Modelling 29(10): 105-119.

https://doi.org/10.1016/S0895-7177(99)00096-5

Itô, K. (1942). On stochastic processes (infinitely divisible laws of probability). PhD. thesis, Japan Journal Mathematics XVIII: 261-301.

Janicki,A., Popova, W. I., Ritchken, P. H. y Woyczynski, W. A. (1997). Option pricing bounds in $\alpha$-stable security market, Communications in Statistics: Stochastics Models 13(4): 817-839. https://doi.org/10.1080/15326349708807453.

Kendall, M. G. y Hill, A. B. (1953). The analysis of economic time-series-part I: Prices, Journal of the Royal Statistical Society. Series A (General) 116(1): 11-34. https://doi.org/10.2307/2980947.

Kruizenga, R. (1956). Put and call options: A theoretical and market analysis, PhD thesis, Massachusetts Institute of Technology.

Landeros Olascoaga, B. E. (2008). ¿Qué está pasando con la contabilidad financiera?, Colegio de contadores públicos de México 1(4):1-5. Consultado el 26 de junio de 2018. www.ccpm.org.mx/avisos/boletines/boletin_noviembreNIFS.pdf

Lévy, P. (1937). Théorie de l'addition des Variables Aléatoires, Gauthier-Villars, Paris.

Mandelbrot, B. (1963). The variation of certain speculative prices, Journal of Business, 36(4): 394-419. http:// dx.doi.org/10.1086/294632. 
Mandelbrot, B. y Taylor, H. M. (1967). On the distribution of stock price differences, Operations Research, 15(6): pp. 1057-1062. https://doi.org/10.1287/opre.15.6.1057.

Mantilla, S. A. (2016). IFRS in Focus. IFRS 9: Instrumentos financieros, Deloitte Touche Tohmatsu Limited. www2. deloitte.com/IFRS in Focus Abril 2016 IFRS 9 Resumen alto nivel.pdf. Consultado el 26 de junio de 2018.

Martínez Palacios, M. T. V., Sánchez Daza, A., y Venegas Martínez, F. (2012). Valuación de opciones americanas: un enfoque de control óptimo estocástico en un horizonte finito con fecha final aleatoria. Análisis Económico, 64(XXVII): 165-183.

McCulloch, J. H. (1978). The pricing of short-lived options when price uncertainty is log-symmetric stable. Working Paper 89, Departament of Economics, Boston College. https://doi.org/10.3386/w0264.

McCulloch, J. H. (1985). Interest-risk sensitive deposit insurance premia: Stable ACH estimates, Journal of Banking and Finance 9(1): 137-156. https://doi.org/10.1016/0378-4266(85)90066-4.

McCulloch, J. H. (1987). Foreign Exchange Option Pricing with Log-Stable Uncertainty, Recent Developments in International Banking and Finance, Lexington, Lexington, pp. 231-245. S. J. Khoury and A. Ghosh eds.

McCulloch, J. H. (1996). Financial applications of stable distributions, Handbook of Statistics, $\quad$ 14: 393-425. https://doi.org/10.1016/S0169-7161(96)14015-3.

McCulloch, J. H. (2003). The risk-neutral measure and option pricing under log-stable uncertainty. Working Paper, Economics departament, Ohio State University.

Merton, R. C. (1973). Theory of rational option pricing. The Bell Journal of Economics and Management Science, 4(1): 141-183. https://doi.org/10.2307/3003143.

Merton, R. C. (1976). Option pricing when underlying returns are discontinuos, Journal of Economic Theory 3(12): 125-144. https://doi.org/10.1016/0304-405X(76)90022-2.

Morales Díaz, J. (2012). Aplicación práctica de la contabilidad de coberturas utilizando opciones, Asociación Española de Contabilidad y Administración de Empresa 7:1-32. Consultado el 26 de junio de 2018. www.aeca.es/ old/faif/articulos/comunicacion7.pdf.

Ortobelli, S. L., Huber, I. y Schwartz, E. S. (2002). Portfolio selection with stable distributed returns, Mathematics Methods of Operations Research, 55: 265-300. https://doi.org/10.1007/s001860200182.

Ortobelli, S. L., Rachev, S. y Schwartz, E. S. (2004). The problem of optimal asset allocation with stable distributed returns, Stochastic Processes and Functional Analysis, 238: 295-347. https://doi.org/10.1007/s001860200182.

Ortobelli, S. L., Biglova, A., Huber, I., Racheva B. y Stoyanov, S. (2005). Portafolio choice with heavy tailed distributions, Journal of Concrete and Applicable Mathematics, 3(3): 353-376.

Osborne (1959). Brownian motion in the stock market, Operations research 7(2): 145-173.

Pelmeneva, A. A. y Talipova, A. S. (2015). Real options as a tool for arctic offshore fields valuation and project management according IFRS 6, Society of Petroleum Engineers - SPE Russian Petroleum Technology Conference. https://doi.org/10.2118/176694-MS.

Popova,W. I. y Ritchken, P. (1998). On bounding options princing in paretian stable markets, The Journal of Derivatives 5(4): 32-43. https://doi.org/10.3905/jod.1998.408001.

Rachev, S. T., Ortobelli, S. L. y Schwartz, E. S. (2004). The problem of optimal asset allocation with stable distributed returns, Stochastic Processes and Functional Analysis Marcel Dekker Inc. 238: 295-347. A. C. Krinik, R. J. Swift eds.

Rodríguez Aguilar, R. y Cruz Aké, S. (2013). Valuación de opciones de tipo de cambio asumiendo distribuciones $\alpha$-estables, Contaduría y Administración, 58(3): 149-172. https://doi.org/10.1016/S0186-1042(13)71225-7.

Rodríguez Díaz, D. del P. (2017). Antes NIC 39 ahora NIIF 9: nuevos desafíos para los contadores, Contabilidad y Negocios, 12(23): 6-21.

Ross, S. A. (1976). The arbitrage theory of capital asset pricing. Journal of Economic Theory, 13(3): 341-360. https://doi.org/10.1016/0022-0531(76)90046-6.

Samuelson, P. A. (1965). Rational theory of warrant pricing, Industrial Management Review 6: 13-31. https://doi.org/10.1007/978-3-319-22237-0_11. 
Scalas, E. y Kim, K. (2006). The art of fitting financial time series with Levy stable distributions, Munich Personal RePEc Archive August (336):1-17.

Sierra Juárez, G. (2007). Procesos de Hurst y movimientos brownianos fraccionales en mercados fractales, Revista de Administración, Finanzas y Economía 1(1): 1-21.

Sprenkle, C. (1961). Warrant prices as indicator of expectation of expectations and preferences, Yale Economics Essays 1(2): 178-231.

Stein, E. M. y Stein, J. C. (1991). Stock price distributions with stochastic volatility: An analytic approach, The Review of Financial Studies 4(4): 727-752. https://doi.org/10.1093/rfs/4.4.727.

Scott, L. O. (1987). Option pricing when the variance changes randomly: Theory, estimation, and an application, The Journal of Financial and Quantitative Analysis 22(4): 419-438. https://doi. org/10.2307/2330793.

Uhlenbeck, G. E. y Ornstein, L. S. (1930). On the theory of Brownian motion. Physical Review, 36: 823-841. https://doi.org/10.1103/PhysRev.36.823.

Venegas Martínez, F. (2005). Bayesian inference, prior information on volatility, and option pricing: A maximum entropy approach, International Journal of Theoretical and Applied Finance 8(1): 1-12. https://doi.org/10.1142/S0219024905002755.

Villeneuve, S. y Zanette, A. (2002). Parabolic A.D.I. Methods for Pricing American Options on Two Stocks. Mathematics of Operations Research 27: 121-149. https://doi.org/10.1287/ moor.27.1.121.341 